\title{
Gramtools enables multiscale variation analysis with genome graphs
}

\author{
Brice Letcher ${ }^{1 *}$, Martin Hunt ${ }^{1,2}$ and Zamin lqbal ${ }^{1 *}$
}

*Correspondence:

bletcher@ebi.ac.uk; zi@ebi.ac.uk

${ }^{1}$ EMBL-EBI, Hinxton, UK

Full list of author information is

available at the end of the article

\begin{abstract}
Genome graphs allow very general representations of genetic variation; depending on the model and implementation, variation at different length-scales (single nucleotide polymorphisms (SNPs), structural variants) and on different sequence backgrounds can be incorporated with different levels of transparency. We implement a model which handles this multiscale variation and develop a JSON extension of VCF (jVCF) allowing for variant calls on multiple references, both implemented in our software gramtools. We find gramtools outperforms existing methods for genotyping SNPs overlapping large deletions in M. tuberculosis and is able to genotype on multiple alternate backgrounds in $P$. falciparum, revealing previously hidden recombination.
\end{abstract}

Keywords: Genome graph, Pangenome, Variant calling, Plasmodium falciparum, Mycobacterium tuberculosis, VCF

\section{Background}

Variant calling, the detection of genetic variants from sequence data, is a fundamental process on which many other analyses rely. There are two standard approaches, each with their own limitations. For Illumina data, mapping to a reference genome causes reference biases that affect discovery and genotyping: mapped reads favour the reference allele and reads in divergent regions fail to map [1-4]. For PacBio/Oxford Nanopore Technology (ONT) data, genomes can be fully assembled, and therefore, the discovery and genotyping problems are in principle partially solved, by aligning each assembly against a reference. (There are caveats about how to get high per-base quality, either by hybrid ONT/Illumina or PacBio Hifi reads, but we leave this aside). However, the problem of how to coherently represent all of the variants in a cohort, comparing all against all, remains challenging both algorithmically and in terms of outputting results.

There are data structures that in principle can genotype alternate alleles which include both long structural variants and SNPs-some implementations include Cortex, GraphTyper, vg, and BayesTyper [4-7]. All of these are based on graph representations of one form or another ranging from genotyping a whole-genome de Bruijn graph

(c) The Author(s). 2021 Open Access This article is licensed under a Creative Commons Attribution 4.0 International License, which permits use, sharing, adaptation, distribution and reproduction in any medium or format, as long as you give appropriate credit to the original author(s) and the source, provide a link to the Creative Commons licence, and indicate if changes were made. The images or other third party material in this article are included in the article's Creative Commons licence, unless indicated otherwise in a credit line to the material. If material is not included in the article's Creative Commons licence and your intended use is not permitted by statutory regulation or exceeds the permitted use, you will need to obtain permission directly from the copyright holder. To view a copy of this licence, visit http://creativecommons.org/licenses/by/4.0/. The Creative Commons Public Domain Dedication waiver (http://creativecommons.org/publicdomain/zero/1.0/) applies to the data made available in this article, unless otherwise stated in a credit line to the data. 
(Cortex), mapping all reads to a whole-genome Directed Acyclic Graph (DAG) of informative k-mers (BayesTyper), mapping all reads to a whole-genome graph of minimising k-mers and matched haplotype index (vg/Giraffe [8]) or remapping premapped reads either to local DAGs of SNPs and indels off the reference (GraphTyper) or to graphs built from structural variant breakpoints (GraphTyper2 [9]). These all reduce the impact of reference bias, and allow cohort genotyping at consistent sites, but all of them struggle with the issue of representation. We highlight two important types of situations which pose representation challenges - any good solution will need to address these, and conversely, solving these alone would address most of the needs of most users.

First, a long deletion that "covers" 10 SNPs will in principle have $2^{10}$ alternate alleles. This would be painful to output in the widely used variant call format [10] (VCF), but more fundamentally, it would force the genotyper to make statements about long alternate alleles that only differ by SNPs. If two long alleles, one true and one not, differed by just one SNP, then under most models they would have very similar likelihoods, and it would be impossible to tell clearly which was the correct allele. In other words, there would be no fine-scale data about variants, making SNP filtering impossible (Additional File 1: Figure S1).

Indeed, there is no tool (to our knowledge) that self-advertises as supporting joint SNP and structural variant (large insertions/deletions) genotyping (although we note the software Birdsuite was developed for jointly genotyping SNPs and copy-number variants [11]). Benchmarking in this paper shows GraphTyper2 and vg both work in this scenario if given VCF input. The second key challenge occurs when variants fall on different genetic backgrounds such as diverged MHC haplotypes [3] or large insertions-for this there is no current solution.

We highlighted above two key situations to address: SNPs as alternatives to long deletions, and SNPs on top of long alternate haplotypes. In both of these cases, variants are bound by relationships. In the first, they are mutually exclusive and in the second, they occur on top of alternative sequence backgrounds, thus combining exclusion and a hierarchy. We call such variation nested and identify these relations as sufficient to capture a valuable proportion of natural genetic variation. We therefore model the genome as a directed acyclic graph that is a succession of locally hierarchical subgraphs. This is a rich enough model to incorporate our key use-cases, without incurring the price of excessive generality; we discuss the benefits and limitations in the "Discussion" section. Based on this, we can use gramtools to identify these nested site relationships, leverage them during genotyping and output variants, genotypes and likelihoods in a file format extending VCF. This is, to our knowledge, the first framework for jointly analysing genetic variation at different scales (SNPs and structural variants) and on different sequence backgrounds.

We start by detailing the genome graph workflow implemented in gramtools and an algorithm for genotyping nested variation. We build graphs of variation from 2498 samples at four Plasmodium falciparum surface antigen genes which harbour high levels of diversity, including two that each have two diverged allelic forms (DBLMSP and DBLMSP2). We use simulated haplotypes from graphs to evaluate the impact of nesting on genotype confidences, and then benchmark with empirical data from $14 \mathrm{P}$. falciparum samples which have high quality PacBio assemblies for ground truth. We then address the two canonical use-cases we highlighted above. We show that gramtools 
outperforms vg and GraphTyper2 when genotyping long deletions and all the overlapping small variants from a cohort of 1017 Mycobacterium tuberculosis genomes. Finally, we apply gramtools to the use-case for which no current solution exists. We genotype 706 African and SE Asian P. falciparum genomes at the gene DBLMSP2, which possesses variation on two diverged backgrounds which had previously appeared to either never or, very rarely, recombine. This generates the first map of genetic variation on both diverged backgrounds, revealing patterns of recombination that were previously unknown.

\section{Results}

gramtools implements a workflow for building, genotyping and augmenting genome graphs (Fig. 1). Genotyping serves two main use cases in this workflow. First, it is used for inferring a sample's closest path in the graph, which we can use as a "personalised" reference genome, since it should be a closer approximation than any individual genome would be. We are thereby able to discover new variants by using standard referencebased variant callers with this personalised reference, an approach previously described in $[3,12,13]$. Second, gramtools is used for genotyping cohorts of samples on a graph containing all such discovered variants. Neither case requires finding variants absent from the graph because novel variants are found by standard tools applied to the personalised reference. Thus, while other tools such as vg [4] perform full alignment of reads to the graph, gramtools achieves the same aims while only needing to do exact matching of reads

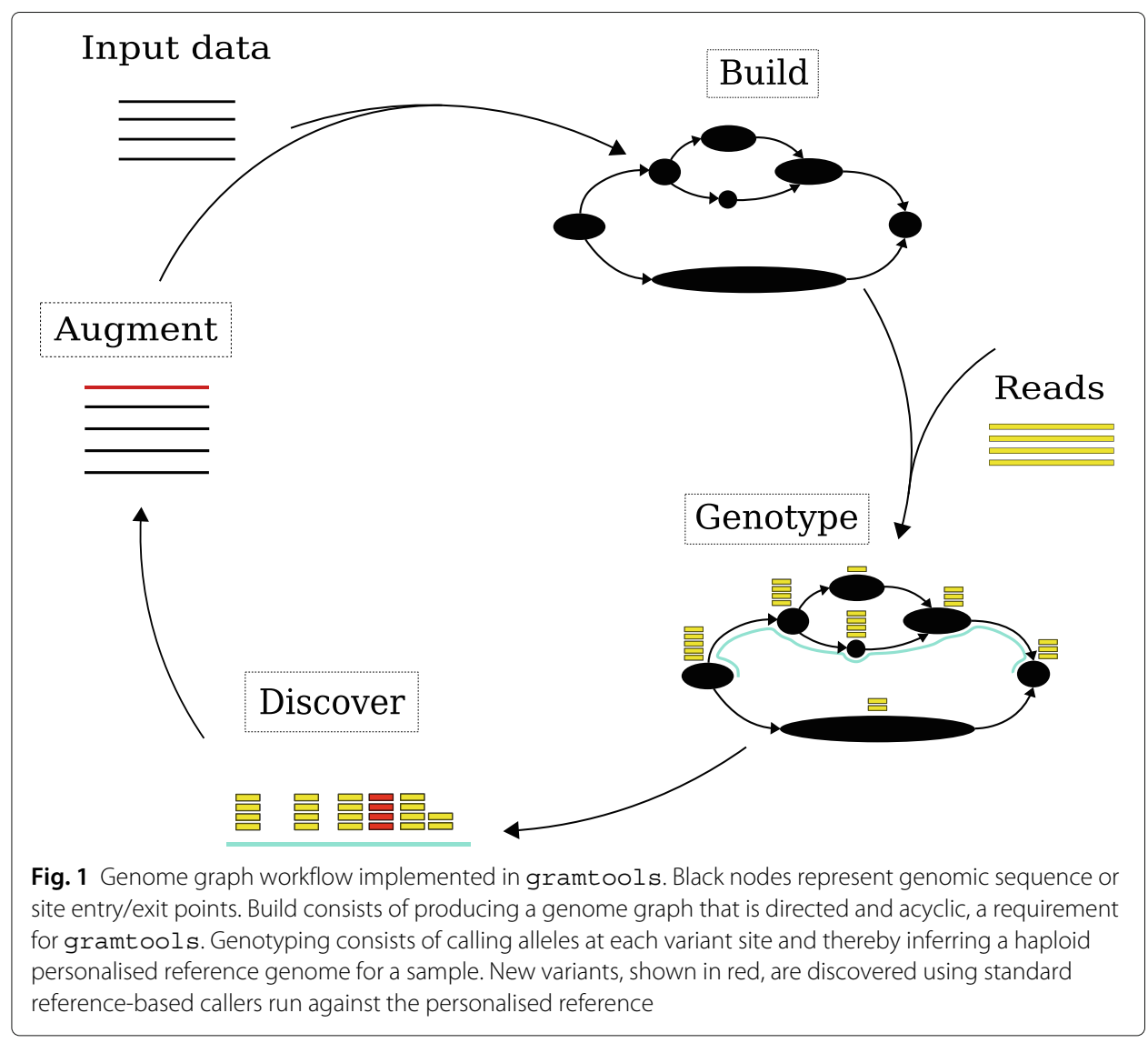


(after quality trimming). Our implementation is currently limited to high-accuracy short reads (e.g. Illumina); support for long erroneous reads requires matching read substrings.

\section{Graph constraints and genotyping with the vBWT}

In gramtools, sequence search in genome graphs is supported using the compressed suffix array [14] of a linearised representation of the graph, which we call variationaware Burrows-Wheeler Transform (vBWT). The vBWT algorithm was introduced with a proof-of-concept implementation in [12]; details of how it converts BWT string matching to graph mapping are provided in the "vBWT data structure in gramtools" section. A key requirement of the $\mathrm{vBWT}$ is that the graph be decomposable into a succession of subgraphs (sites) each of which is strictly nested (see Fig. 2 and the "Graph definitions" section for formal definitions) interspersed by linear, non-variable regions.

This results in a graph where genotyping a site with alternate alleles is well-defined, preserving a notion with biological value, but also places some restrictions on the structure of the genome graph. We give an example in Additional File 1: Figure S3 showing a pair of allowed/disallowed graphs that generate equivalent sequence.

These graphs can be built from multiple sequence alignments (MSAs) or a reference genome plus VCF file; we used MSAs in this paper. The construction process, first introduced in [15], is explained further in the "Genome graph construction and make_prg" section, and we consider the implications of this model in the "Discussion" section. The original vBWT implementation was slow and did not support nesting [12]. In this paper, we introduce our nesting implementation and have optimised the codebase to improve mapping, coverage recording and genotyping.

\section{Genotyping nested genome graphs}

gramtools genotypes a nested DAG in which variant sites have been defined (see the "Graph definitions" section). Sites are genotyped independently, choosing the maximumlikelihood allele under a coverage model that draws on ideas from kallisto [16],

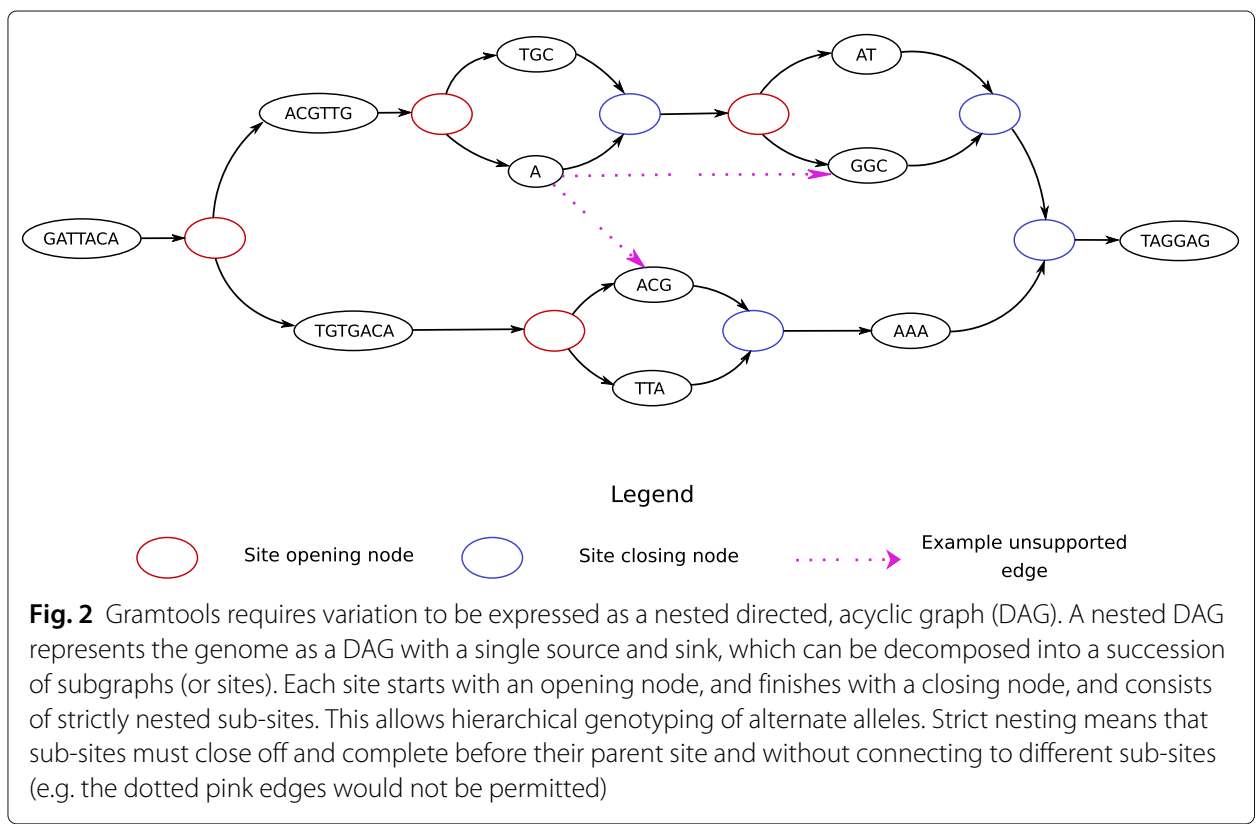


including both per-base coverage information, and equivalence class counts for reads that could equally support different alleles (see the "Genotyping model" section). Genotype likelihoods are calculated for each allele at a site, and the ratio of likelihoods between the maximum likelihood allele and the next best is termed the genotype confidence. The personalised reference (PR) genome inferred by gramtools is the path obtained by taking the maximum-likelihood call at each variant site, and the genotype confidence at each site provides a measure of the adequacy of the inferred PR. For example, a stretch of lowconfidence calls suggests no close path in the graph was found or that no reads mapped in this region. While the genotyping model can handle both haploid and diploid cases, in the diploid case two unphased PRs are produced (as was done in [3]) whereby the two alleles at heterozygous sites get randomly allocated to each. In this paper, we evaluate gramtools on haploid organisms only.

With nested variation, we apply the genotyping model recursively from child sites to their parents, with candidate alleles in parent sites generated based on the genotype calls of child sites (see the "Nested genotyping" section). We refer to each outgoing branch from a parent site as a haplogroup; if there is no nesting then these correspond to alleles, but when there is nesting, they can be seen as alternate sequence backgrounds (see Fig. 3 in the following section)

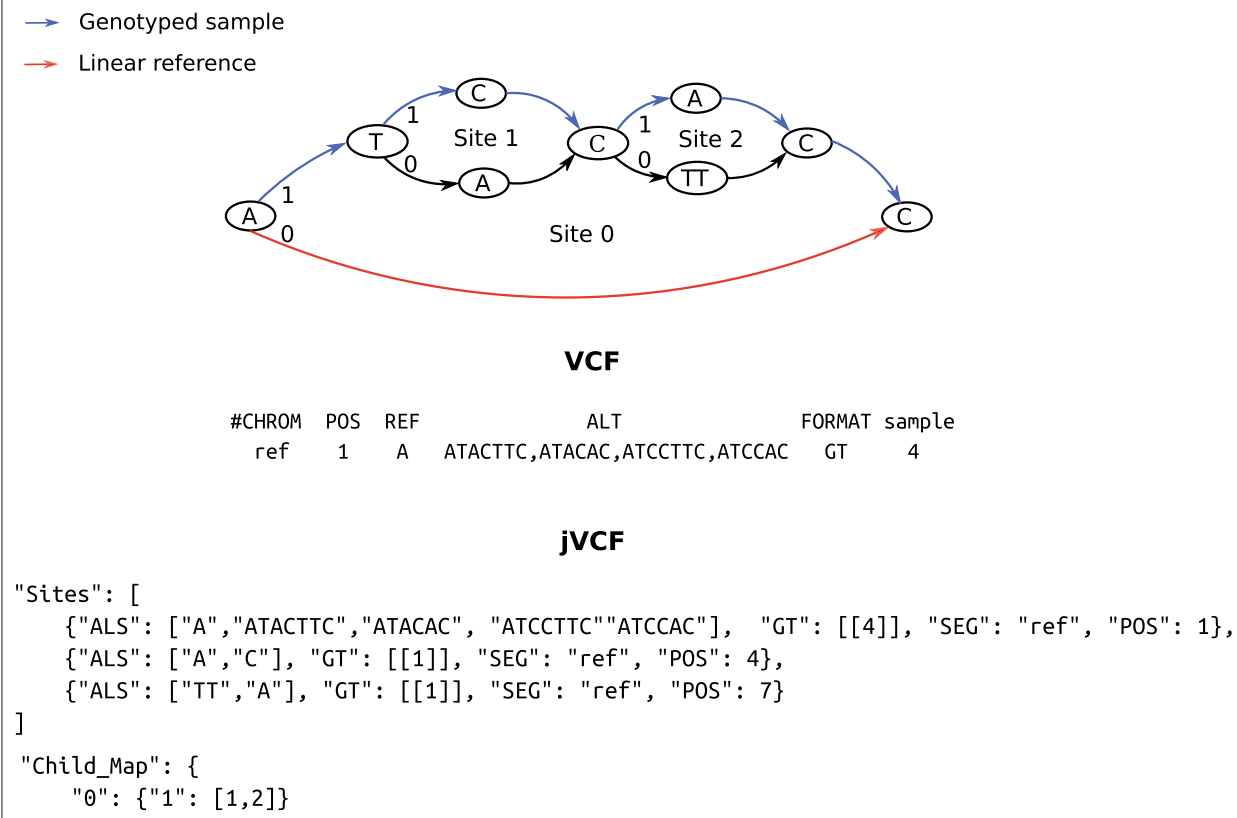

VCF

\section{jVCF}

"Sites": [

["Als": ["A", "ATACtTC", "ATACAC", "ATCCTTC" "ATCCAC"], "Gt": [[4]], "SeG": "ref", "pos": 1\}, \{"ALS": ["A", "C"], "GT": [[1]], "SEG": "ref", "POS": 4\},

Fig. 3 Example VCF and jVCF calls for a graph containing nested variation. Three sites exist in the graph, and site-opening edges are labelled by haplogroup ( 0 or 1). Below the graph, parts of VCF and jVCF files describe a genotyped sample (blue path in the graph). In VCF, only one variant site is genotyped, with the "sample" column stating that allele 4 has been called (this corresponds to "ATCCAC", as the numbering is 0-based). However, the two nested variants - sites 1 and 2 - cannot be expressed independently from site 0 , as they do not occur on the linear reference (red path in the graph). jVCF stores the same information as VCF in the "Sites" array (one entry per site) and additionally records site relationships using a "Child_Map" entry, which states that sites 1 and 2 occur under site 0 (first key), haplogroup 1 (second key). This places sites 1 and 2 on an alternate reference background (the sequence "ATACTTC") allowing them to be expressed in the "Sites" entry. Alternate reference sequences are obtained by following haplogroup 0 at each nested variant site (here this spells "ATACTTC") 


\section{jVCF output format}

In attempting to provide genotypes at all variant sites within a cohort, one is inevitably faced with densely clustered genetic variants, which leads to difficulties when using VCF. First, the genomic positions of output records can overlap, implying they should be considered jointly. Second, sites can occur on sequence backgrounds (haplogroups) that do not include the reference genome sequence. In VCF, overlapping records require careful genotyping and alternate references are not supported. To address these limitations, gramtools outputs a variant call file in JavaScript Object Notation (JSON), a widely used format for storing data as key-value pairs [17]. The format stores variant records mirroring VCF and additionally stores genome graph-specific information-we thus call it jVCF.

To illustrate the purpose of jVCF, we show in Fig. 3 a graph containing nested variation and parts of VCF and jVCF files describing genotype calls in this graph. Both formats store the reference and alternate alleles of a variant site and its location in the genome and can genotype the first site (site 0 ) in the graph. However, sites 1 and 2 occur on a different sequence background from the linear reference and thus cannot be expressed independently of site 0 in the VCF output. In jVCF, the parent/child site relationships are stored in a "Child_Map" entry which locates variant sites based on what sequence background (haplogroup) they fall on. This enables jVCF to express sites 1 and 2, by placing them on a different reference background with its own coordinates and to give independent genotypes for those sites. In jVCF, storing site relationships also makes incompatibilities between sites explicit, allowing variants in overlapping genomic positions to be consistently genotyped (see the "Nested genotyping" section).

A full format specification for $\mathrm{jVCF}$ is provided in Additional File 1. In addition to a jVCF file, gramtools outputs a regular VCF file containing only non-nested sites, yielding a VCF file with no overlapping records and referring only to the linear reference genome.

\section{Validation of nested genotyping with simulated data}

Our first simulation was designed to evaluate both genotyping performance and whether nested genome graphs resulted in improved calibration of genotype confidences. We based the simulation on a real example where there are two alternate haplotypes each bearing variants, building graphs of $P$. falciparum variation for two genes, DBLMSP and DBLMSP2. These genes exhibit a dimorphism at the Duffy Binding-Like (DBL) domain, each having a region $>500 \mathrm{bp}$ in length with two allelic forms that are highly diverged $[18,19]$. We built two versions of the graph, one without any nesting, and one allowing nesting up to five levels deep (see the "Genome graph construction and make_prg" section). The graphs were built from high confidence variant calls in 2498 samples from the Pf3k project [20] (see the "Graph construction" section for details). The graph without nesting contained 451/413 variant sites for DBLMSP/DBLMSP2 respectively and the graph with nesting contained 558/500 variant sites respectively. The nested graphs contain more sites because they allow SNPs/indels to occur on different sequence backgrounds, as illustrated in Fig. 4. We randomly sampled 10 paths from the non-nested graph (which therefore exist in the nested one), recorded the implied truth variant calls, simulated reads from the paths and passed them to gramtools for genotyping (see the "Path and read simulation" 


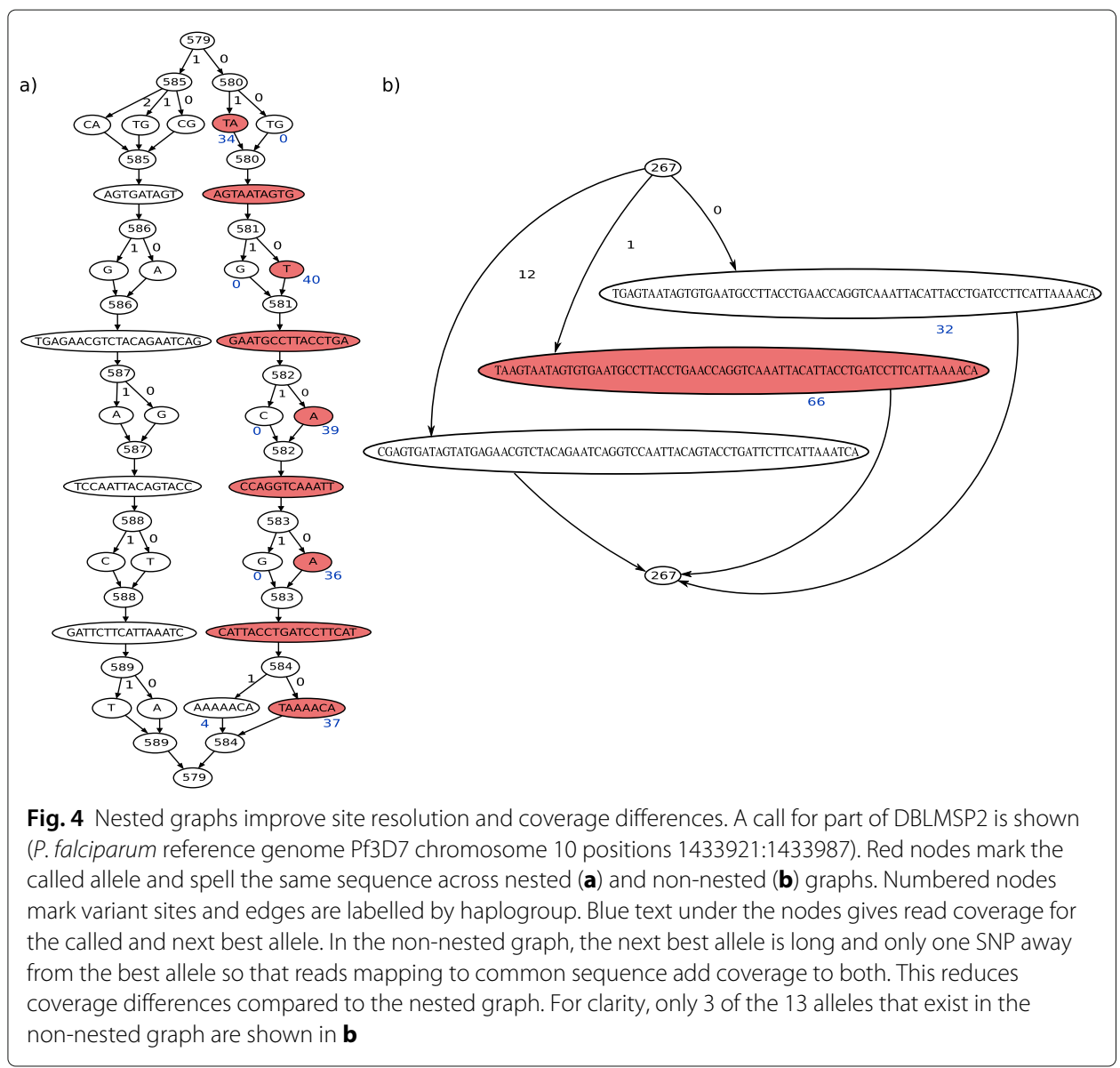

section for details). Out of 17,280 evaluated calls in the non-nested graph, gramtools recovered 99.9\% (recall) and of all calls made, 99.8\% were correct (precision). In the nested graph, out of 21,160 evaluated calls, recall and precision were both $99.9 \%$.

While gramtools genotypes both types of graphs to high accuracy, nested graphs provide better call resolution. This is shown in Fig. 4 for (a subregion of) DBLMSP2 where the nested graph reflects the allelic dimorphism: SNPs/small variants fall on top of each allelic form. We also confirm that the genotype confidence of correct calls is also increased, since nesting allows likelihood calculations based on coverage precisely at SNPs on alternate haplotypes, rather than an average across the whole haplotype (coverage shown in Fig. 4, and effect on confidences shown in Additional File 1: Figure S6).

\section{Benchmarking gramtools genotyping against single-reference variant callers at surface antigens}

Building on the simulation results in P. falciparum, we set out to evaluate gramtools genotyping in comparison with standard single-reference callers SAMtools [21] (the classical "pileup" variant caller) and Cortex [5] (which discovers bubbles in a de Bruijn graph, and then maps flanking sequence to the reference to get coordinates). We include Cortex because it has previously been shown to produce high-quality calls even in the indel-rich P. falciparum genome and can successfully identify alternate alleles at the genes 
we analyse here [22] but has no capacity to consider nested variation. We built a wholegenome graph containing variation from 2498 P. falciparum samples (as in the simulation experiment) in four surface antigen genes: DBLMSP, DBLMSP2, EBA175, and AMA1 (see the "Graph construction" section). The additional genes, EBA175 and AMA1, are both vaccine targets, where there is great value in being able to correctly identify known and novel variation $[23,24]$. We used 14 P. falciparum validation samples with both Illumina data and high-quality PacBio long-read assemblies [25], and which had been excluded from graph building, to assess gramtools genotyping. Performance is measured as the edit distance between the gene sequence with called variants applied and the long-read assembly, normalised by gene length. Note that the gramtools graphs are themselves built from Cortex calls in the 2498 samples (see the "Graph construction" section); thus, we are comparing genotyping via a graph of known population variation (gramtools) with reference-based variant calling (SAMtools and Cortex).

We show in Fig. 5 the scaled edit distance (i.e. edit distance divided by gene length) achieved by these tools on the 14 validation samples, aggregated across all four genes. As a baseline, we show the distribution of scaled edit distances between the 3D7 (reference) gene sequences and the truth assemblies, giving a mean distance of $3.7 \%$ (top-left panel, dotted line). SAMtools and Cortex both improve on this, achieving means of $2.3 \%$ and $1.3 \%$ respectively. gramtools outperforms both variant callers, achieving a mean of $0.6 \%$. We provide performance for each individual gene in Additional File 1: Figure

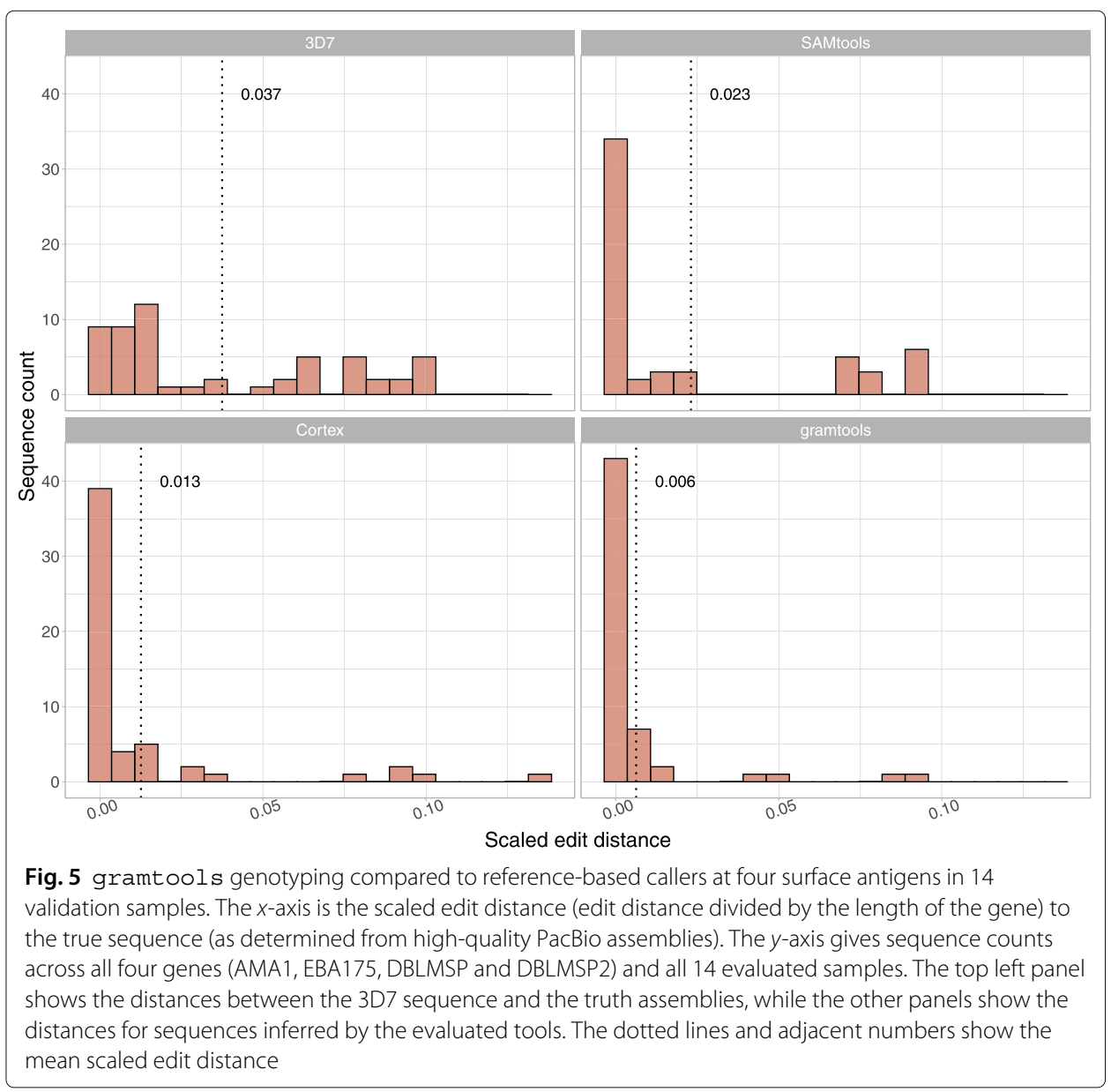


S7-10 and further confirm gramtools genotyping finds optimal paths in the graphs in Additional File 1: Figure S11.

\section{Application: unified SNP and large deletion analysis in M. tuberculosis}

Nested variation occurs naturally when jointly genotyping small variants overlapping structural variants. We assessed how gramtools compares to two other genome graph tools, GraphTyper2 [9] and vg [4], in one such situation in M. tuberculosis. We evaluated each tool's ability to genotype a fixed set of input small variants (SNPs and indels) and overlapping deletions.

We started from variant calls obtained by running Cortex on 1017 publicly available Illumina samples (see the "Variant discovery" section). We also produced high-quality hybrid assemblies for 17 of these samples using matched Illumina [26] and PacBio reads [27] (see the "Hybrid assembly of the 17 evaluated samples" section). These assemblies were used as ground truth for evaluating genotyping.

We identified 73 high quality large deletion calls in the 17 samples, spanning a total of 45 distinct genomic regions (confirmed using the assemblies (see the "Variant discovery" section)). We then extracted all variation in the 1017 samples overlapping these 45 regions. Together, these provide the variant sites at which we evaluate each tool.

For gramtools, we built a genome graph of each deletion region from multiplesequence alignments of the Cortex variant calls applied to the M. tuberculosis $\mathrm{H} 37 \mathrm{Rv}$ reference genome [28] (see the "gramtools genome graph construction" section). The graphs were then combined with the rest of the reference genome. To genotype the same variants in GraphTyper2 and vg, we merged the VCF files of all 1017 input samples using bcftools. VCF is the required input format for genotyping in GraphTyper2 and the only input format that worked in vg after failing on multiple sequence alignments (see the "vg and Grapht Typer2 genome graph construction" section).

We first looked for each of the 73 known deletions in each tool's VCF output and found GraphTyper2 called all 73, gramtools called 70 and vg called 66 . We then assessed each tool's ability to resolve each deletion region in the 17 evaluation samples. For each region, we applied called variants to the $M$. tuberculosis reference genome and measured edit distance to the truth assembly using minimap2 [29] (similar results were obtained using bowt ie2 [30]; see Additional File 1: Figure S17 and the "Mapping evaluated regions to truth assemblies" section).

Figure 6 shows the cumulative distribution of scaled edit distances (edit distance divided by the length of the aligned sequence) for each tool. gramtools achieves the lowest mean distance to the truth (1.2\%), followed by vg(2.4\%) and GraphTyper2 (3.2\%) (as a baseline, the mean distance of the reference genome sequence to the truth is $4.8 \%$ ). We note that without 8 long, false positive insertions with edit distances $>0.5$, the mean distance for GraphTyper2 is $2.6 \%$. gramtools also achieves the highest fraction of perfectly resolved sequences (edit distance 0) (86.7\%), followed by GraphTyper 2 (69.3\%) and vg (54.0\%). A small number of sequences remained unmapped (9 for gramtools, 12 for GraphTyper2 and 13 for vg (Additional File 1: Figure S18)).

To understand genotyping performance in more detail, we broke down called variants into different types (insertions, deletions, SNPs) and sizes and measured precision (what proportion of calls made were correct) and recall (what proportion of the expected 


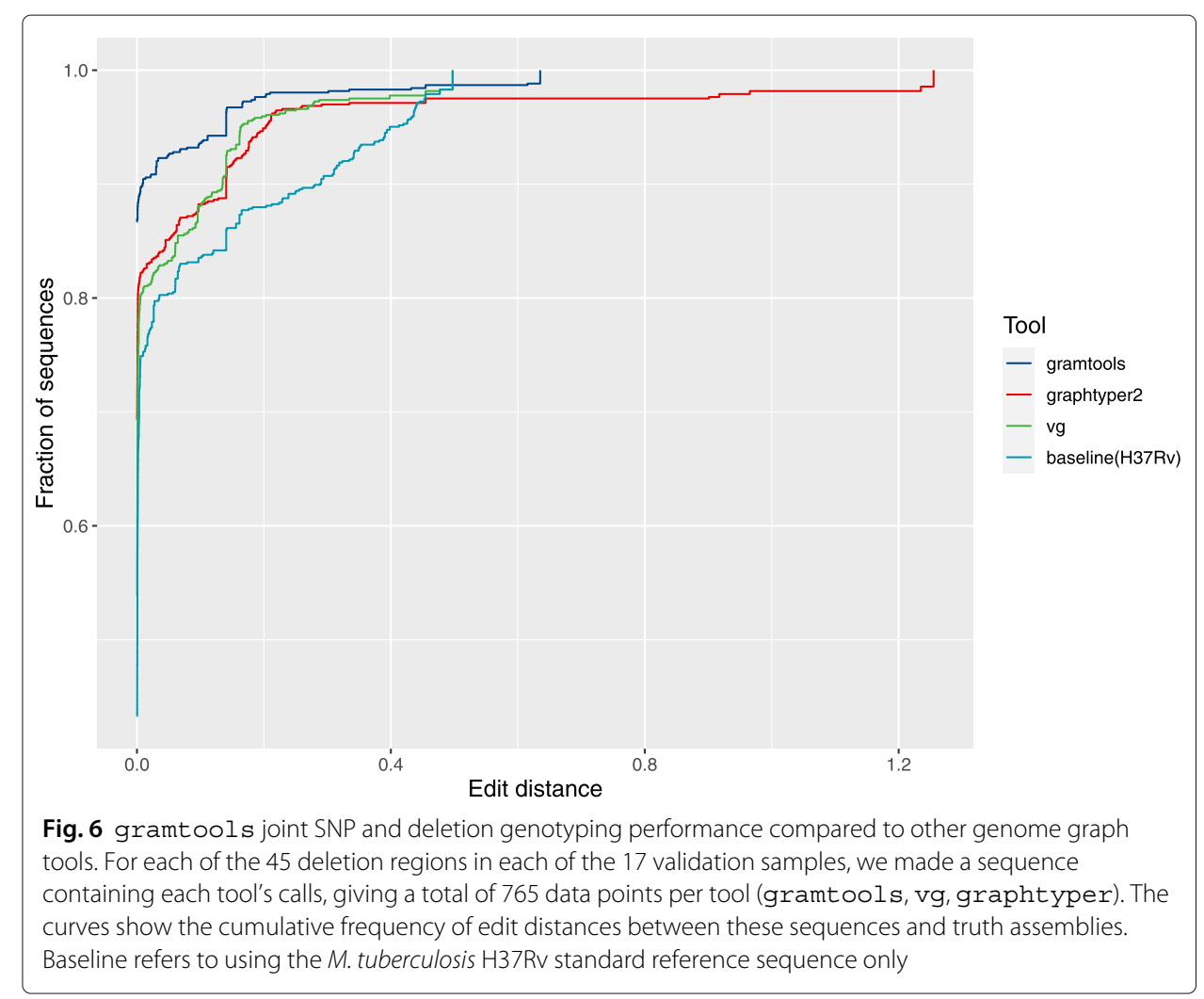

calls were recovered) (see the "Evaluating variant calls using varifier" section). Compared to the other tools, we found vg has a larger number of incorrect and missing small variants (insertions and deletions < 10bp, and SNPs). Notably, SNP recall and precision were $57.2 \%$ and $86.7 \%$ for vg, compared to $91.3 \%$ and $93.8 \%$ for gramtools and 90.0\% and 99.1\% for GraphTyper2 (Additional File 1: Figure S19). Similarly, we found GraphTyper2 has a larger number of incorrect and missed large insertions and deletion calls (> 50bp): for large deletions, GraphTyper2 recall and precision were $67.3 \%$ and $64.4 \%$, compared to $97.8 \%$ and $99.6 \%$ for gramtools, and $97.1 \%$ and $99.5 \%$ for vg (Additional File 1: Figure S19). gramtools achieved the highest recall across all variant categories but has lower precision than vg or GraphTyper2 for some categories, notably

Table 1 Computational performance of each tool

\begin{tabular}{llllll}
\hline & Index & \multicolumn{3}{l}{ Map and genotype } \\
\hline & Disk (Mb) & Max RAM (Mb) & Speed (sec) & Max RAM (Mb) & Speed (reads/sec) \\
vg & 29 & 609 & 105 & $605[158]$ & 3,961 \\
gramtools & 153 & 480 & 32 & 632 & 34,290 \\
GraphTyper2 & - & - & - & $869[88]$ & 7,604 \\
\hline
\end{tabular}

Index: genome graph processing step allowing subsequent read mapping. For vg, includes a graph pruning step to reduce graph complexity (else temporary disk use exceeded $500 \mathrm{~Gb}$ before completion, see "vg and Grapht Typer2 genome graph construction" section). GraphTyper2 has no separate indexing operation. Map and Genotype: Speed shows the average number of reads mapped across the 17 samples (10.7 million) divided by the average CPU time. vg and GraphTyper 2 have separate read mapping and genotyping steps: for speed, CPU time is summed, and for RAM, mapping is shown followed by genotyping in square brackets. GraphTyper2 does not implement its own mapping but requires an input file of reads mapped to a linear reference genome; mapping RAM and speed is shown for bowtie2 with default parameters. metrics: Mb, megabytes; sec, total CPU seconds (accounts for multi-threading, 10 threads used for genotyping in each tool) 
SNPs and small (1-10bp) and mid-size (11-50bp) insertions (Additional File 1: Figure S19).

In terms of computational performance, gramtools processed the most reads per CPU second while using comparable amounts of RAM on this dataset (Table 1). A bottleneck in vg is temporary disk use, exceeding 500 Gigabytes without pruning the graph to remove densely clustered variation. For GraphTyper2, we include counting a separate mapping step to the reference genome (with bowtie2) as it is a prerequisite to genotyping (for vg and gramtools, performance includes mapping reads to genome graphs before genotyping). While gramtools' mapping and genotyping is 4 to 8 times faster than vg and GraphTyper2 in this benchmark, we are also aware of gramtools' much lower mapping speed to the human genome [31]. Computational performance depends on the genome and variants under analysis and on the genome graph approach; we consider these further in the "Discussion" section.

In this experiment, GraphTyper 2 and vg are able to genotype variation at multiple scales. One caveat is the VCF file they genotype can contain inconsistencies. For example, if one VCF record describes a deletion and another describes an overlapping SNP, a reference call at the deletion and an alternate call at the SNP are inconsistent because the two calls imply different sequences. This occurs because the variants are related but expressed in isolation. By contrast gramtools models site relationships explicitly, outputting a VCF file without inconsistencies and a jVCF file mapping the nested variation.

An output format like our proposed jVCF becomes especially important when analysing more complex variation such as SNPs on top of alternate haplotypes, where variants need to be expressed against different references. We now show such an application of multiscale variation analysis using the P. falciparum surface antigen DBLMSP2, which would not be possible using the VCF files output by vg or GraphTyper2.

\section{Application: charting SNPs on top of alternate haplotypes}

When two diverged forms of a gene segregate in a population, we want to access small variants on top of each. Returning to the surface antigen DBLMSP2 in P. falciparum - which we have shown is accurately genotyped by gramtools using simulated and real data-we assessed whether gramtools' multiscale genotyping and jVCF output could recover the two diverged forms of DBLMSP2 and access variation on top of each form. We genotyped 706 P. falciparum samples from Ghana, Cambodia and Laos using gramtools and analysed a combined jVCF file of all calls in all samples (see the "P. falciparum dimorphic variation analysis" section). Genotyping, including read mapping, used an average of 1.14 Gigabytes of peak RAM, processing an average of 2525 reads per CPU second.

Figure 7 shows a matrix of calls in each sample at each variant site inside the DBL domain of DBLMSP2, known to be dimorphic [32]. The tree on the left depicts a hierarchical clustering of the sample haplogroups. Its most basal split distinguishes two forms of the domain (form 1 (dark pink) and form 2 (light pink) on the right of the heatmap), with an average scaled edit distance between the two forms of $16.8 \%$ compared to within-form distances of $1.4 \%$ and $4.8 \%$. gramtools can thus recover two divergent forms, as expected given the known dimorphism. Building a phylogenetic tree of the sequences confirmed the presence of the two forms and showed high concordance with the clustering tree in how samples are assigned to each form (Additional File 1: Figure S20). 


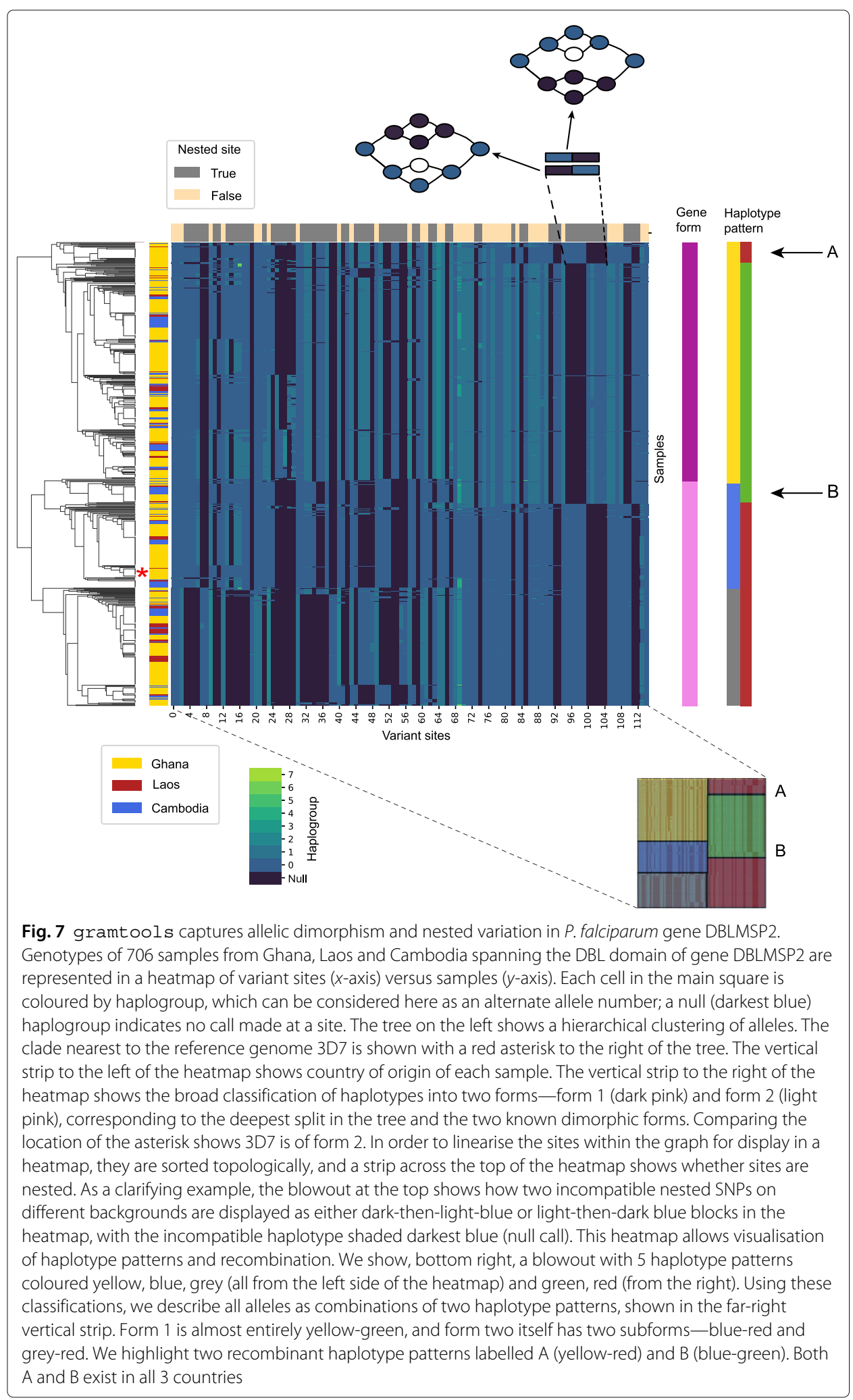


gramtools also produces calls in nested sites, shown as dark bars at the top of the matrix. As an example, we provide an illustrative blowout at the top of the heatmap of a region where variants are nested and occur on different sequence backgrounds. The graphs illustrate how the nested sites are called mutually exclusively: when one of the gene forms has a genotype call, the other form receives a null call (shown as the darkest blue-coloured cells), and vice-versa. This is essentially showing that there are SNPs on both genetic backgrounds (the dimorphic types) and gramtools is genotyping variants irrespective of background.

Interestingly, the heatmap in Fig. 7 also shows, for the first time to our knowledge, clear evidence of recombination between the two forms of DBLMSP2. At the bottom-right of the figure, we show a blowout of the matrix that is coloured by broad haplotype patterns, with form 1 being predominantly yellow followed by green and form 2 being predominantly blue or grey followed by red. This reveals two sets of samples (labelled A,B by the blowout) which are inter-form recombinants. Those labelled A have a yellow haplotype (form 1 ) followed by red (form 2) and those labelled B have blue (form 2) followed by green (form 1).The leftmost column of the matrix is coloured by the country of origin of each sample. Strikingly, for each group of samples (i.e. A and B) almost identical recombinants exist across each of Ghana, Laos and Cambodia. We do not yet know if these inter-form recombinants derive from a single recombination event (either ancestral to the samples or transmitted across countries) or if this recombination event has occurred multiple times independently.

Finally, we indicate, using a red asterisk on the right of the tree, the closest clade to the reference genome sequence 3D7. Leveraging gramtool s' graph model and jVCF output, we can move beyond a 3D7-only reference-based analysis that would neither genotype nested variants on the two different backgrounds nor reveal the inter-form recombinants.

\section{Discussion}

Genetic variation occurs through different mechanisms at different scales ranging from SNPs to large structural variants. The need to jointly analyse SNPs and structural variants therefore arises immediately when trying to genotype a cohort. We have presented a method for identifying, calling and outputting such variation in gramtools. By identifying site relationships in the graph, gramtools is able to genotype incompatible sites mutually exclusively and to output variation both against the standard reference genome and against locally defined alternate references.

One of the challenges of extending linear references with graphs is recognising modelling assumptions. Working from a single reference implicitly assumes that individuals within a species have genomes that are close to the reference. When addressing this model's limitations by moving to a graph, we are forced to make new modelling choices. At one end of the spectrum is the gramtools approach: genome graphs must be nested, directed acyclic graphs. This simple model allows direct access to two key notions we want to use: easily distinguishing horizontal (paralog-like) and vertical (ortholog-like) variation and defining distinct alternate sequence backgrounds. At the other extremes are very general sequence graphs with no ordering, whether De Bruijn (which collapse all repeats of size k) or vg-like (bidirected and allowing cycles). These models better support 
more complex events such as duplications and inversions, with an added cost in complexity of implementation (e.g. reconstructing variant sites, identifying coordinates and mapping reads).

Choice of graph model also affects the computational performance of read mapping. Data structures supporting linear-time exact matching exist for a restricted class of graphs (see Wheeler graphs [33], which include De Bruijn graphs). In gramtools, graphs do not have this property, and we use a data structure (the vBWT) with run time and memory use that can scale exponentially with genome size and density of stored variation. In practice, however, gramtools shows good computational performance on the $P$. falciparum and M. tuberculosis graphs used in this study. Here, we analyse variation from a few thousand samples and a small number of regions, but gramtools has also successfully been used to genotype 70,000 (whole genome) M. tuberculosis samples in a graph containing 1.25 million variants (one variant every 3.5 bases) [34]. We are also, however, aware of gramtools' very low mapping speed on the human genome [31]. More work is required in the pangenomics field to understand the different real-world performance challenges of repetitiveness (P. falciparum is much more repetitive than human), genome size (microbes are tiny but diverse) and amount, type and density of variation.

Applying gramtools' genome graph model on microbial datasets, we obtain three main results. First, in $P$. falciparum genes with high diversity, gramtools genotyping with genome graphs outperforms reference-genome-based callers. Second, gramtools provides superior genotyping accuracy compared to genome graph tools vg and GraphTyper2 when jointly genotyping large deletions and overlapping small variants in M. tuberculosis. (We note that during the finalisation of this paper, a new caller based on vg named Giraffe [8] was released, which we have not tested here). Third, we show how locally defined alternate references allow accessing small variants on top of diverged forms of a dimorphic gene in P. falciparum.

These results highlight three central concepts for genome graph based analyses: compatibility, consistency and interpretability.

First, while genome graphs extend beyond a single linear reference, maintaining compatibility with linear references is essential. gramtools outputs variation in terms of the standard reference genome in a VCF file and also produces a personalised reference genome, allowing reference-based callers to discover previously inaccessible variation. Many genomic analyses rely on a linear reference, which provides a simple coordinate system for referring to genomic annotations and comparing individuals. Recently, the rGFA format for describing genome graphs was proposed [35]; starting from a central linear reference, it assigns stable names and offset coordinates to alternate references. rGFA is a valuable and complementary idea to the jVCF described here: it assigns coordinates and references in constructed genome graphs, while our jVCF describes sites and called variation in genotyped genome graphs. Like the graphs used by gramtools, rGFA works on globally linear graphs in order to maintain clear homology relationships. Although this feature is not implemented, jVCF could easily be extended to store rGFA-defined alternate references, allowing for expressing variant calls against any reference.

Second, genome graphs offer the opportunity to genotype cohorts of samples consistently. By representing all variation found in a set of samples, they can be used to produce a full sample-by-site matrix. gramtools achieves this by detecting all variant sites in 
the graph and outputting them, along with their relationships, in a jVCF call format. Previous work has explored graph decomposition into a fixed set of variant sites [36] and is available in vg with the deconstruct command. However, vg genotyping currently does not output all such sites nor define and output alternate references. gramtools provides (to our knowledge) the first working implementation of consistent graph to variant site mapping.

An important determinant of compatibility and consistency is the graph construction process. In gramtools, we use our tool make_prg [15]. From a multiple sequence alignment, make_prg collapses common sequence between samples, clusters the remaining sequence into subgroups, and repeats the process recursively. This algorithm provides two main advantages. First, it limits recombination to similar input haplotypes, which reduces combinatorial explosions in variant dense regions, a source of computational bottlenecks and graph ambiguity [37]. Second, it naturally creates a hierarchy between sites as they are gradually defined on different sequence backgrounds. This captures incompatibility between sites (as in SNPs under a large deletion) as well as the process of divergent sequence evolution.

Finally, while single references and VCF provide good interpretability, we show how analysing two diverged forms of a dimorphic surface antigen in P. falciparum (DBLMSP2) benefits from locally defined alternate references. In contrast to existing sequences such as the alternate MHC loci in the human reference genome [38], here these are tied together in a graph-based framework. Outputting variation on different sequence backgrounds can provide finer resolution than with a single reference and will enable studying the functional impact and population genetics of nested variants.

\section{Conclusions}

We provide a framework for identifying and genotyping multiscale variation in genome graphs and show its successful implementation in gramtools. We find good genotyping performance compared to state-of-the-art genome graph tools GraphtTyper2 and vg and additionally provide an analysis of allelic dimorphism using multiple references which to our knowledge can only be performed by gramtools.

Multiscale variation analysis goes hand in hand with the gradual extension of reference genomes beyond their linear coordinates. Accessing this complex variation requires careful genome graph construction and stable names and coordinates for referring to alternate references. It also calls for new developments in variant call output formats, a proposal of which we implement and use in gramtools.

\section{Methods}

\section{Graph definitions}

Here, we formally define a variant site and the type of graph that gramtools can support. Let $G=(V, E)$ be a directed acyclic graph (DAG) with a unique minimal and unique maximal element, i.e. $G$ has a unique source and unique sink. Each node $v$ has a number of ingoing edges $\operatorname{deg}^{-}(v)$ and a number of outgoing edges $\operatorname{deg}^{+}(v)$. Define a node $v$ to be opening if $\operatorname{deg}^{+}(v)>1$ and closing if $\operatorname{deg}^{-}(v)>1$. Note that a node can be both opening and closing.

Let $s$ be the sink of $G$. Given any opening node $v$, let $S$ be the set of nodes that are in every path from $v$ to $s$, excluding $v$ itself. Then, $S$ is non-empty because $s$ belongs to $S$. Let $a$ and 
$b$ be any elements of $S$. Then, by definition of $S$, there exists a path that contains both $a$ and $b$. Therefore, using the partial order defined by the edges of $G, a$ and $b$ are comparable and it follows that $S$ is a totally ordered finite non-empty set. Therefore, $S$ contains a unique minimal element, which we denote $c(v)$. Informally, $c(v)$ can be thought of as the first node that "closes" all paths from $v$. Similarly, given a closing node $u$, we define $o(u)$ to be $c(u)$ applied to the transpose of G. Informally, $o(u)$ is the node that "opens" $u$. See Additional File 1: Figure $S 2$ for an example of how $S$ and closing nodes are identified.

We use the notion of opening and closing nodes to define a variant site as follows.

Definition. Let $G$ be a DAG with a unique source and unique sink. A variant site is defined as the subgraph of $G$ induced from $\{u, c(u)\}$ or from $\{o(v), v\}$, where $u$ is any opening node and $v$ is any closing node of $G$.

We remind the reader that for any DAG $G$, there exists at least one ordering of all the nodes $v_{0}, v_{1}, \ldots, v_{n}$ such that given any edge $\left(v_{i}, v_{j}\right)$ of $G, v_{i}$ appears before $v_{j}$ in the ordering. This is called a topological ordering of $G$. Using the above definitions, we can now define the type of graph that is supported by gramtools, which we call a "nested directed acyclic graph".

Definition. Let $G$ be a DAG with a unique source and unique sink. $G$ is said to be a nested directed acyclic graph (NDAG) if there exists a topological ordering of all nodes $v_{0}, v_{1}, \ldots, v_{n}$ such that adding brackets to this ordered list of nodes according to the following rules results in balanced opening and closing brackets:

1 For each opening node $u$, add $[u \text { after } u \text {, and add }]_{u}$ before $c(u)$;

2 For each closing node $v$, add $[v \text { after } o(v) \text { and add }]_{v}$ before $v$, unless these brackets were already added by case 1 .

Note that each matching pair of brackets in the above definition corresponds to one variant site. See Additional File 1: Figure S3 for an illustration.

To be able to index with the vBWT, gramtools would apply the following modifications to the graph, producing a new graph where there is a one-to-one correspondence between the set of opening and closing nodes. Specifically, this means that a node is either opening or closing and cannot open or close more than one node. Essentially, the method entails adding a new node to the graph for each balanced bracket that was added to the topological ordering of the nodes. Starting from the innermost brackets, for each matching pair of brackets $[a \text { and }]_{a}$, where node $a$ precedes $[a$ and node $b$ follows $]_{a}$ in the topological ordering with balanced brackets (so we are considering $\left.\ldots, a,[a, \ldots,]_{a}, b, \ldots\right)$ :

- Add a node called $[a$ with no sequence and an edge $(a,[a)$ to the graph and move the outgoing edges of a to [a;

- Add a node called $]_{a}$ with no sequence and an edge (]$\left._{a}, b\right)$ to the graph and move the incoming edges of $b$ to $]_{a}$.

See Additional File 1: Figure S4 for an illustration of this process.

In practice, gramtools does not need to transform an NDAG or verify if an input DAG is an NDAG, as it takes as input constructed graphs that are already indexable NDAGs. This is achieved using one of two ways described below. 


\section{Genome graph construction and make_prg}

gramtools can construct genome graphs without nested variation from a reference genome and a VCF of variants. Overlapping records in the VCF file are merged by enumerating all possible combinations up to a specifiable limit. This method creates an NDAG because no variant sites overlap, giving a natural balanced bracket representation of sites. However, this approach rapidly fails in variant-dense regions or for large cohorts of samples due to a prohibitively large number of allele combinations. We solve this problem by allowing for nested variation. To build nested graphs, we apply an algorithm called recursive collapse and cluster (RCC) starting from a multiple-sequence alignment. RCC was first introduced in the context of bacterial pan-genomic tool pandora [15] and is implemented in and available at https://github.com/iqbal-lab-org/make_prg.

RCC identifies invariant regions of a given minimum size and collapses them into a single graph node. The remaining regions form variant sites, and each gets clustered based on their $k$-mer content. This procedure is repeated recursively on each cluster, until either a maximum nesting level is reached or the sequences are too small (in which case they are directly enumerated as alternative alleles). In this way, variants appear in subsets of samples with similar sequence backgrounds. The RCC algorithm generates hierarchically nested sites by construction: each cluster of sequences corresponds to one variant site, the clustering process generates distinct clusters, and recursive sequence collapsing occurs fully inside of a cluster, making new clusters nested. RCC thus produces an output graph that is an NDAG. We provide an illustration of RCC and how it induces the balanced bracket representation of an NDAG in Additional File 1: Figure S5.

Two command-line parameters affect what graph gets produced. First, max_nesting is the maximum number of collapse and cluster recursions to perform, which gives the maximum number of nesting levels in the graph. Second, min_match_length is the number of invariant bases between samples for them to be collapsed in a single node. Sequence collapse is what allows paths coming before and after to cross; a larger value thus reduces recombination between the input haplotypes. This provides a way to control combinatorial path explosions in the graph.

\section{vBWT data structure in gramtools}

The vBWT data structure marks variant sites with numeric identifiers so that alleles get sorted and queried together in the suffix array (Fig. 8a). This representation induces branching at each site entry and exit such that mapping has worst-case exponential runtime. To speed mapping, we seed reads from an index storing the mapped intervals of all sequences of a given size $k$. Linear-time exact match indexes on genome graphs exist (e.g. GCSA [39]) but require a prefix-sorting step that is worst-case exponential.

vBWT's numeric identifiers are also used for recording mapped read coverage along variant sites (Fig. 8b). Coverage recording handles two types of uncertainty: horizontal, where sequence is repeated across the genome, and vertical, where sequence is repeated in alleles of a site. To handle horizontal uncertainty, we randomly select one read mapping instance, as is typically done in standard aligners [30]. To handle vertical uncertainty we store allele-level equivalence class counts which are counts of reads compatible with groups of alleles, an idea introduced in kallisto [16]. This allows allelic uncertainty to be accounted for during genotyping. Per-base coverage is also stored on the graph (Fig. 8) and used during genotyping. 
a)

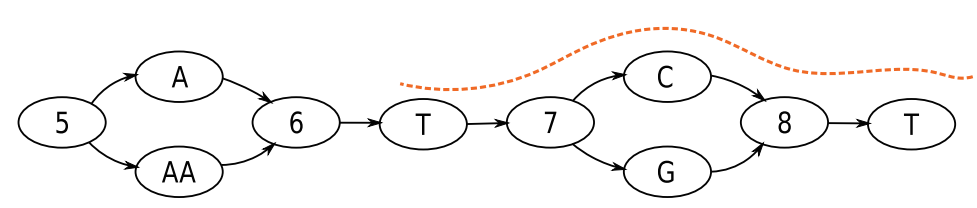

mapping

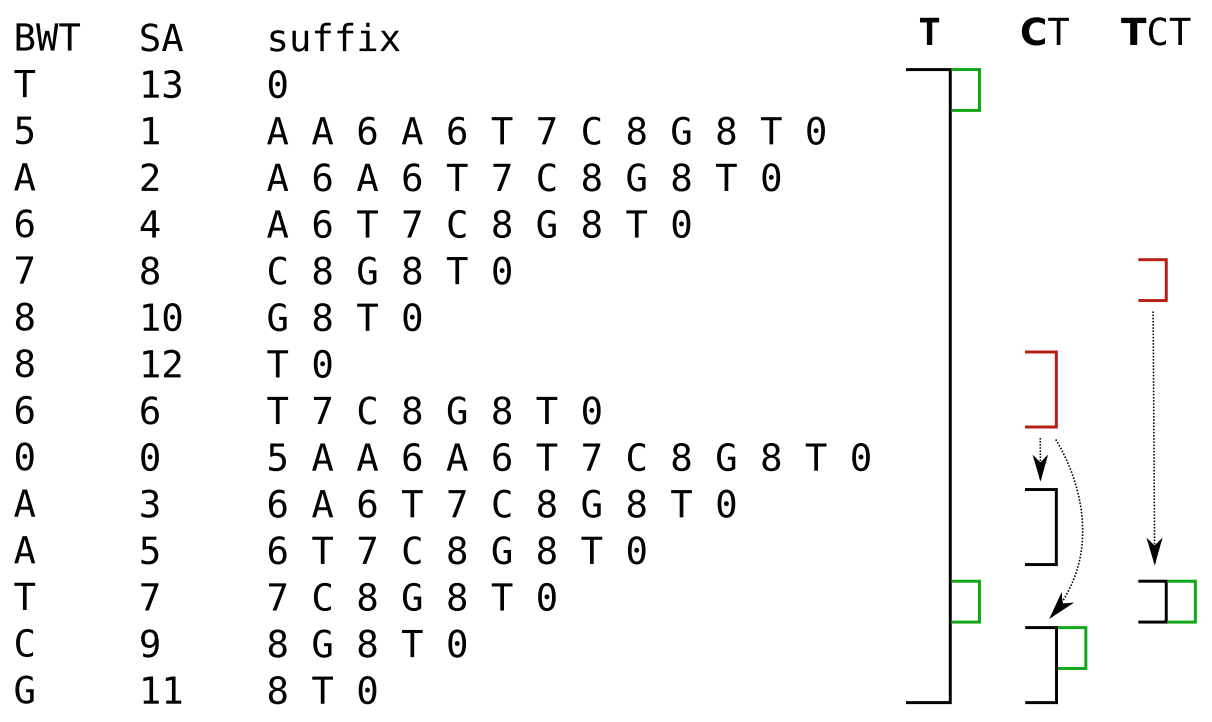

b)

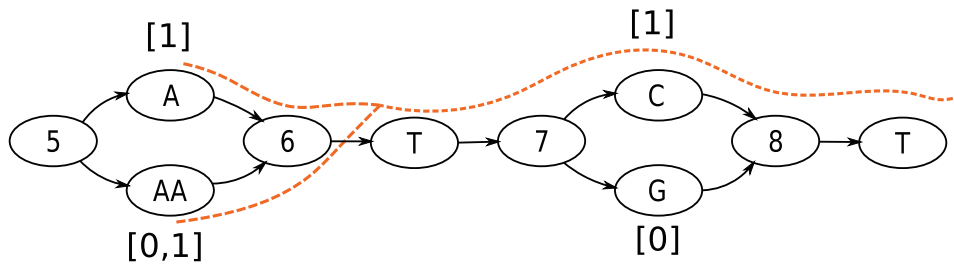

$$
\begin{array}{cc}
{[\{\{0,1\}: 1\},} & \{\{1\}: 1\}] \\
\text { Site } 5 & \text { Site } 7
\end{array}
$$

Fig. 8 gramtools mapping and coverage recording. a Variant-aware Burrows Wheeler Transform (vBWT). Each row of the text matrix encodes one position in a linear representation of the graph. BWT: stores the character in the previous position; SA: suffix array, stores the position in the text; suffix: stores the text from SA position to the end. Two markers are used for every variant site in the genome graph: odd markers mark site entry and even markers allow alleles to sort and be queried together. Black intervals mark regular BWT backward searching, with each match to the currently mapped base shown in green. Arrows from red intervals mark vBWT-specific jumps in and out of sites, making the search branch. The read being mapped is shown in dashed orange. $\mathbf{b}$ Square brackets under allele nodes show per-base coverage storage. Another array shown below stores allele-level coverage at each site. Mapped reads increment equivalence class counts representing compatibility: in this example, the read is compatible with both alleles 0 and 1 at site 5 and only with allele 1 at site 7 . Both kinds of coverage are used in genotyping 


\section{Genotyping model}

The genotyping model in gramtools supports haploid and diploid genotyping. It assigns a likelihood to each candidate allele (or pair of alleles for diploid) computed from base-level and allele-level read coverage.

gramtools stores coverage in equivalence classes, following ideas from [16]. Let $A$ be the set of alleles at a variant site. We partition the set of all reads overlapping $A$ into subsets, i.e. equivalence classes, where all reads belonging to one subset map perfectly to the same subset $X$ of $A$ (e.g. reads that map uniquely to allele 1 , or reads that map equally well to alleles 1 and 2; see Fig. 8b). For each equivalence class, we store a count $c_{X} \in \mathbb{N}$ of reads compatible with the alleles in set $X$, and for each mapped read, we increment its $c_{X}$ at each overlapped site. If a read has multiple (horizontal) mapping instances, we select one at random, and the counts $c_{X}$ are incremented as above. When a read's count $c_{X}$ is incremented, for each allele $a \in X$, the count of each base the read mapped to is also incremented. Base-level counts are written $c\left(a_{i}\right)$, where $a_{i}$ is the $i$ th base of allele $a$.

Coverage compatible with allele $a$ of length $l_{a}$ is defined as $c(a)=\frac{1}{l_{a}} \sum_{i=1}^{l_{a}} c\left(a_{i}\right)$ and incompatible coverage as $i(a)=\sum_{X \subset A: a \notin X} c_{X}$. In this way for each candidate allele, we capture a per-base correct coverage generation process as well as an incorrect coverage generation process on incompatible alleles.

We model the expected per-base read coverage in a site using an estimate of the mean $\lambda$ and the variance $\sigma^{2}$ of true coverage across all variant sites. For each site, true coverage is estimated as the average per-base coverage of the allele with the most coverage. If $\sigma^{2} \leqslant \lambda$, we model observed coverage as coming from a Poisson distribution:

$$
p(c(a)=k \mid \lambda)=e^{-\lambda} \frac{\lambda^{k}}{k !}
$$

Else, we use the negative binomial (NB) distribution

$$
p(c(a)=k \mid p, r)=\left(\begin{array}{c}
k+r-1 \\
k
\end{array}\right)(1-p)^{r} p^{k}
$$

When using the NB distribution, we need to estimate the standard parameters of the NB distribution, $r$ and $p . r$ is estimated from rearranging the formula for the expected variance of NB as $r=\frac{\lambda^{2}}{\sigma^{2}-\lambda}$, and similarly $p$ is estimated from the expected mean of NB as $p=\frac{\lambda}{\lambda+r}$.

We model incorrect coverage $i(a)$ as coming from sequencing errors with rate $\epsilon$ : $p(i(a)=k \mid \epsilon)=\epsilon^{k} . \epsilon$ is estimated from the mean base quality score in the first 10,000 processed reads.

We also use per-base coverage to penalise gaps in coverage. Given a function $g(a)$ returning the number of zero-coverage positions in allele $a$, the probability $p(g(a)=k)$ of seeing $k$ gaps if $a$ is the true allele is $p\left(z e r o \_c o v\right)^{k}$, where $p\left(z e r o \_c o v\right)$ is obtained by computing $p(c(a))$ using the above formula with $c(a)$ set to zero. In practice, we use $\frac{k}{l_{a}}$ as the exponent instead of $k$ so as not to penalise long alleles.

These three terms combined give the likelihood of allele $a$

$$
\mathcal{L}(a)=p(c(a)) p(i(a)) p(g(a))
$$


The allele $a^{\prime}$ that gets called is the maximum-likelihood allele, and we define the genotype confidence of the call as

$$
\min _{a \in A: a \neq a^{\prime}} \frac{\mathcal{L}\left(a^{\prime}\right)}{\mathcal{L}(a)}
$$

which is the likelihood ratio of the called allele and the next most likely allele.

This holds for haploid genotyping. For higher ploidy, the likelihood function generalises to a set of alleles $S$ as

$$
\mathcal{L}(S)=p(i(S)) \prod_{a \in S} p(c(a)) p(g(a))
$$

where $i(S)=\sum_{X \subset A: X \cap S=\emptyset} c_{X}$.

For diploid genotyping, $S=\left\{a_{1}, a_{2}\right\}$ and $p(c(a))$ is parameterised by $\frac{\lambda}{2}$ because we expect half the total site coverage on each of two called alleles.

\section{Nested genotyping}

The gramtools genotyping model is applied recursively from child sites to their parent sites. Calls in child sites restrict the set of alleles considered in the parent so that the number of choices is reduced: for each outgoing path from a site, $\prod_{i}^{n} p_{i}$ paths are considered, where $p_{i}$ is the number of distinct alleles called at site $i$ (e.g. in diploids 0,1 or 2 ) and $n$ the number of child sites encountered. Some extra paths are also retained when genotype confidence for a child site is low, in order to propagate uncertainty to parent calls. If there are more than 10,000 possible alleles, only the 10,000 most likely alleles are considered. This does not require enumerating all possible alleles as the most likely alleles in child sites have already been computed.

An example of the nested genotyping procedure is shown in Fig. 9. To maintain coherence, if child sites on two different branches of a parent site are genotyped, whole branches can get invalidated. For example at a ploidy of one if an outgoing branch from a parent site is called, all children sites on the other branches receive null calls.

\section{P. falciparum surface antigen graphs and genotyping validation Graph construction}

We started from VCF files produced by running Cortex, a de novo assembly-based variant caller [5], on read sets of 2,498 samples from the Pf3k project [20] (all reads are publicly available on the ENA, see the Availability of data and materials section). Cortex has a very low false positive call rate and can call the divergent forms of P. falciparum surface antigens [22]. The Cortex independent workflow was run, using the bubble caller with $k=31$. The VCF files are publicly released on zenodo (see Availability of data and materials) and Cortex is publicly available at https:/github.com/iqbal-lab/cortex.

For each surface antigen gene (DBLMSP, DBLMSP2, EBA175 and AMA1), we generated sequences for each sample by applying Cortex variants at the gene coordinates, plus 5000 bp on each side, to the P. falciparum 3D7 reference genome. We generated multiplesequence alignments of each gene using mafft [40] and passed them as input to our construction tool make_prg. For the simulation experiment, two graphs were built for DBLMSP and DBLMSP2 with maximum nesting levels of 1 and 5. The graphs without nesting have 451 and 413 variant sites for DBLMSP and DBLMSP2, and the graphs with nesting have 558 and 500 variant sites respectively. 


\section{Path and read simulation}

From each non-nested graph, 10 paths were simulated and threaded through the nested graph using gramtools' simulate command. This results in jVCF files recording the true genotypes for each path in each graph. Illumina HiSeq25 75-bp reads $(0.023 \%$ perbase error rate) were simulated from each unique path using ART [41] at 40-fold coverage, genotyped using gramtools and calls evaluated by comparing the genotyped and truth jVCF files.

The nested graph contains more paths than the non-nested graphs due to allowing greater recombination between variant sites. We therefore simulated paths from the non-nested graph to ensure each path exists in both graphs.

\section{Comparison with reference-based callers}

The nested graphs of the four surface antigens DBLMSP, DBLMSP2, EBA175 and AMA1 were combined in positional order with the rest of the reference genome, using a custom script (see the Availability of data and materials section).

For each of the 14 validation samples, we ran SAMtools and Cortex using the $P$. falciparum 3D7 reference genome and gramtools using the surface antigen genome graph. For each genotyped sample, gramtools infers a haploid personalised genome

a)

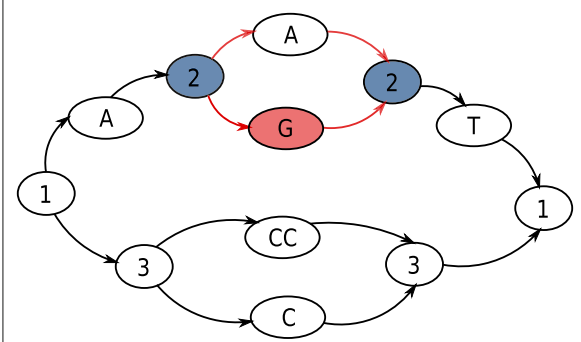

Ref: A Alts: $\{A, G\} \quad G T: G$

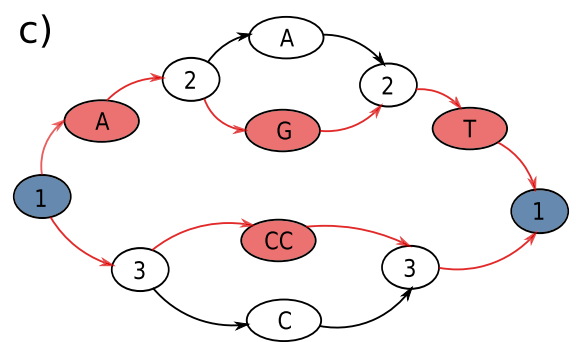

Ref: AAT Alts: $\{A G T, C C\} \quad G T: A G T$ b)

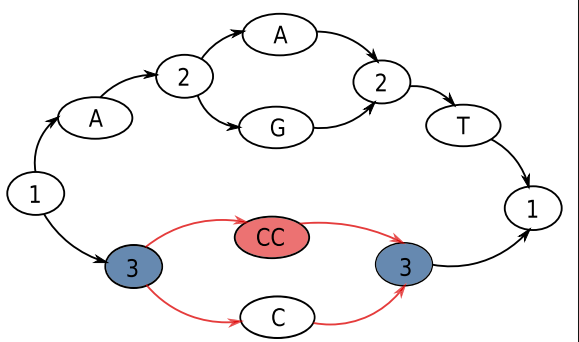

Ref: CC Alts: $\{C C, C\}$ GT: CC

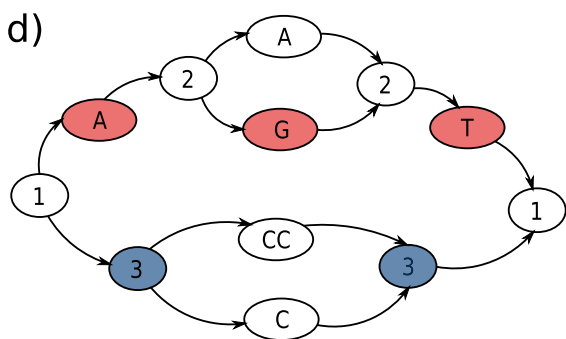

Ref: CC Alts: \{\} GT: null

Fig. 9 Nested genotyping procedure. Nodes with numbers mark the start and end of variant sites. In each panel, blue-filled nodes mark which site is being processed, red-filled nodes mark called alleles, and red paths mark alleles considered for genotyping. Ref is the reference allele, Alts are the alleles considered for genotyping, and GT is the called genotype. The example shows haploid genotyping. a Genotyping of child site 2. Allele "G" gets called. b Genotyping of child site 3. Allele "CC" gets called. c Genotyping of parent site 1. Alts are generated from the alleles called in child sites 2 and 3 : allele " $G$ " is used from site 2, and allele " $C C$ " is used from site 3, producing alleles "AGT" and "CC". Allele "AGT" gets called, going through site 2. d In c, the path going through site 2 was called. Because genotyping is haploid, the call at site 3 is invalidated (GT becomes "null") 
$(\mathrm{PR})$ as the whole-genome path taking the called allele at each variant site. SAMtools and Cortex were then run once more using the personalised reference instead of 3D7.

When mapping the gene sequence with variants applied to the truth assemblies, we measure performance as the edit distance reported by bowtie2 (version 2.4.1) divided by gene length.

\section{M. tuberculosis SNP and large deletion analysis Hybrid assembly of the 17 evaluated samples}

Each sample was initially assembled using Unicycler [42] and Canu [43], followed by Circlator [44] using the corrected reads output by Canu. Unicycler version 0.4.8 was used with the option '-mode bold', Illumina reads given using the options '- short 1 and '- short2', and the PacBio subreads using the '- long' option. Canu version 2.0 was used with the option 'genomeSize $=4.4 \mathrm{~m}$ ' and the PacBio reads provided with the option '-pacbio-raw'. The only exception was sample N1177, which was initially assembled using Flye [45] version 2.8-b1674 with the PacBio subreads input with the option '-pacbio-raw'.

The initial assembly for each sample was chosen for further manual polishing based on inspection of mapped reads and comparison with the H37Rv reference genome. The Unicycler assembly was used for samples N0004, N0091, N0155, N0157, N1283, N0072, N1202 and N0153. The Canu assembly was used for samples N0031, N1176, N0052, N0136, N0146, N1216, N1272 and N0054. Redundant and/or contamination contigs were removed from samples N0072, N1202, N0052, N0136, N0146, N1216 and N1272. Manual fixes were applied to samples N0054 and N0153 by breaking contigs at errors, with the aid of the Artemis Comparison Tool (АСT) [46], and re-merging using Circlator using the default settings. Next, Pilon [47] (version 1.23) was run iteratively on each assembly using the Illumina reads as input, mapped with BWA-MEM [48] (version 0.7.17-r1188, default settings) until no more corrections were made, up to a maximum of 10 iterations. Finally, the 'fixstart' function of Circlator was used to ensure that each assembly began with the $d n a A$ gene, for consistency with the H37Rv reference genome. The result was all 17 samples assembled into a single, circularised contig.

\section{Variant discovery}

We first obtained variant calls from the read files of the 17 evaluated samples and an additional 1000 samples available in the ENA (see the Availability of data and materials section). We ran Cortex to obtain the calls, using our lab's wrapper clockwork version 0.8.3, publicly available at https://github.com/iqbal-lab-org/clockwork. clockwork runs Cortex's independent workflow using the bubble caller with $k=31$. The VCF files are publicly released on zenodo (see Availability of data and materials).

Cortex identified a total of 73 deletions in the 17 evaluated samples, between 100 and 13,000 bases in length and falling in 45 distinct genomic regions. To validate the calls, we mapped their corresponding long-read assemblies to the $M$. tuberculosis H37Rv reference genome with minimap2, which validated 68 . The remaining 5 were manually confirmed using ACT: for each sample we mapped the short reads to the reference genome and to the assembly using bowtie2 and mapped the assembly to the reference using nucmer [49]. In $\mathrm{ACT}$, we view all three together and validate a deletion when it appears in the 
assembly-reference mapping at the expected coordinates and when read pileups confirm the event. These are shown in Additional File 1: Figure S12-16.

Having validated all the deletions, we extracted all Cortex calls occurring under the 45 deletion regions in the 1017 samples, giving us a joint set of large deletions and overlapping SNPs and indels.

gramtools genome graph construction

We built one genome graph for each of the 45 regions identified as containing large deletions in our 17 evaluation samples. As for the P. falciparum surface antigen graphs, for each region, we applied Cortex calls to the M. tuberculosis H37Rv reference genome, generated multiple sequence alignments with mafft, passed them as input to our construction tool make_prg and combined them with the rest of the genome.

vg and Grapht Typer2 genome graph construction

We set on building a vg genome graph from the same multiple sequence alignments (MSA) used by gramtools to maximise comparability. Using vg version 1.26.0, we built each of the 45 regions from MSA using vg construct and combined them with the invariant parts of the $M$. tuberculosis $\mathrm{H} 37 \mathrm{Rv}$ reference genome using vg concat.

Indexing this graph, a prerequisite to read mapping and variant calling, used $>10$ Terabytes of temporary disk space before we stopped it. We deemed $>500$ Gigabytes prohibitive and set that as a limit. We ran vg prune to remove densely clustered variation from the graph and, after exceeding 1 Terabyte of disk indexing the pruned graph using default parameters, successfully indexed the pruned graph with parameters $-\mathrm{k} 10-\mathrm{X} 3$.

We then ran vg call for each of our 1017 samples against the MSA graph. However, after successful mapping to this indexed graph, $\mathrm{vg}$ call failed with a segmentation fault.

We therefore built a graph from a VCF file instead. We ran vg deconstruct - $p$-e to obtain a VCF file describing the variants identified by vg in the vg MSAconstructed graph, and manually validated the variation using one sample when compared to gramtools. However, running vg construct with this VCF also failed with a segmentation fault.

We therefore used vg graph construction and genotyping from a merged VCF of all variants in the 45 regions which we produced using bcftools. This ran successfully after graph pruning to stay under our disk limit.

This VCF file was also used as input to GraphtTyper2 via graphtyper version 2.5.1, running its genotype_sv subcommand. GraphtTyper2 only accepts VCF files as input and not MSAs.

\section{Covered positions and number of variants}

Altogether, the 45 deletion regions cover 51,701 bp of the reference genome. The variants under them cover 4105 reference positions in 1,109 sites in the gramtools graph and 2,386 positions in 1434 sites in the merged VCF file used by vg and GraphTyper2.

\section{Mapping evaluated regions to truth assemblies}

We evaluated a total of 3,060 sequences by mapping them to truth assemblies: 17 samples $x 45$ regions $x 4$ tools (gramtools, vg, Grapht Typer 2 and the reference genome sequence). Using bowt ie2, $10.4 \%$ all sequences failed to be fully aligned due to excessive divergence between the called sequence and the truth. 
To recover more alignments, we used minimap2 which is designed to align more highly diverged sequences (such as ONT long reads) [29]. For each evaluated sequence, we took the alignment with the greatest number of matches to the assembly and extracted assembly sequence of the same length from the first aligned position (including soft- or hard-clipped). We obtained the edit distance between the two sequences from Needleman-Wunsch alignment using edlib [50]. Using this approach reduced the proportion of unaligned sequences to $1.1 \%$.

To ensure evaluated alignments are unambiguous, we filter them by MAPQ $\geq 30$ so that the probability they are non-unique is $\leq 10^{-3}$ as estimated by minimap2. This removed $0.62 \%$ of the evaluated sequences. For each tool, 13 of 765 sequences were not mapped or had insufficiently high mapping quality. The number of unmapped and low MAPQ sequences for each tool are shown in Additional File 1: Figure S18.

We required the VCF records output by each tool to have a FILTER status set to "PASS". This changed results only marginally, giving the same number of unmapped and low MAPQ sequences and a decreased mean edit distance by $0.11 \%$ for GraphtTyper2, $0.08 \%$ for gramtools and no differences for $\mathrm{vg}$.

Evaluating variant calls using varifier

varifier is a tool for measuring accuracy of variant calls in a VCF using a reference genome and a truth assembly. Given a variant call, varifier determines if it is correct by aligning the reference genome sequence with called variant applied (plus some flanking sequence to make the alignment specific) to the truth assembly. To compute call precision, which we define as the fraction of calls made that are correct, this procedure is applied to each variant in the evaluated VCF file. To compute call recall, which we define as the fraction of calls made out of all expected calls, varifier first aligns the reference genome to the truth assembly to derive a set of expected variant calls. Each expected call is then evaluated (by the mapping procedure above) against an 'induced truth genome' obtained by applying the evaluated VCF call's variants to the reference genome sequence. Then, if the expected call has been made in the evaluated VCF, it will be found in the induced truth genome. For both recall and precision, we restricted the evaluation to calls with FILTER set to "." or "PASS" in order to ignore low-confidence calls. This led to an improvement in recall and precision of $0 \%$ and $1.9 \%$ (gramtools), $0.1 \%$ and $2.1 \%$ (graphtyper 2 ) and $0.9 \%$ and $1.1 \%(\mathrm{vg})$ on average across the variant types.

varifier is described in more detail in [51] and available at https://github.com/iqballab-org/varifier.

\section{P. falciparum dimorphic variation analysis}

We took a subset of 706 samples from Ghana, Cambodia and Laos out of the 2498 samples used to build the $P$. falciparum genome graphs (see the "Graph construction" section). Using gramtools, each sample was genotyped using the graph containing four surface antigens (DBLMSP, DBLMSP2, EBA175, AMA1). We combined each sample's jVCF output file into a single multi-sample jVCF file using gramtools' combine_jvcf executable and analysed the final jVCF using custom scripts (see the Availability of data and materials section). 


\section{Supplementary Information}

The online version contains supplementary material available at https://doi.org/10.1186/s13059-021-02474-0.

Additional file 1: Contains all supplementary text and figures for this paper.

Additional file 2: Review history

\section{Acknowledgements}

The authors thank Rachel Colquhoun for the algorithms and first development of make_prg, Sorina Maciuca for the vBWT data structure and algorithms behind gramtools and Robyn Ffrancon for software engineering in gramtools. We would also like to thank the anonymous reviewers, whose detailed feedback led to considerable improvements in the manuscript.

\section{Peer review information}

Anahita Bishop was the primary editor of this article and managed its editorial process and peer review in collaboration with the rest of the editorial team.

\section{Review history}

The review history is available as additional file 2 .

\section{Authors' contributions}

BL developed gramtools, performed the analyses, drafted the manuscript and wrote the repository for the study (see the Availability of data and materials section). MH produced the M. tuberculosis assemblies and Cortex VCF files, helped using ACT and developed varifier. Zl designed the study and produced the P. falciparum Cort ex VCF files. MH and $\mathrm{ZI}$ defined the graph constraints and edited the manuscript. MH and ZI designed, and BL modified, the genotyping model. All authors reviewed and approved the manuscript.

\section{Funding}

BL is funded by an EMBL predoctoral fellowship. MH is funded by the Wellcome Trust/Newton Fund-MRC Collaborative Award [200205] and the Bill \& Melinda Gates Foundation Trust [OPP1133541]. Open Access funding enabled and organized by Projekt DEAL.

\section{Availability of data and materials}

gramtools is open-source under an MIT license and publicly available on Github [52] (https://github.com/iqbal-laborg/gramtools). The version of gramtools used in this paper is archived on Zenodo [53].

We provide an open repository for reproducing all results in this study, available at https://github.com/iqbal-lab-org/ paper_gramtools_nesting. The repository README provides all the instructions and commands to obtain the data and to re-run each part of the analysis (using Snakemake [54]). The data used are all openly available. Accessions for data deposited at the ENA are stored as tables, listed in https://github.com/iqbal-lab-org/paper_gramtools_nesting\#inputdata. All other data, as well as a software container, are available on zenodo at https://doi.org/10.5281/zenodo.5075458 All versions/commits of the software used in this study are frozen in the software container and can be found at https:// github.com/iqbal-lab-org/paper_gramtools_nesting/blob/master/container/singu_def.def.

\section{Declarations}

\section{Ethics approval and consent to participate}

Not applicable.

\section{Competing interests}

The authors declare that they have no competing interests.

\section{Author details}

${ }^{1}$ EMBL-EBI, Hinxton, UK. ${ }^{2}$ Nuffield Department of Medicine, University of Oxford, Oxford, UK.

Received: 10 February 2021 Accepted: 20 August 2021

Published online: 06 September 2021

\section{References}

1. Brandt DYC, Aguiar VRC, Bitarello BD, Nunes K, Goudet J, Meyer D. Mapping bias overestimates reference allele frequencies at the HLA genes in the 1000 genomes project phase I data. G3: Genes Genomes Genetics (Bethesda, Md.) 2015;5(5):931-41. https://doi.org/10.1534/g3.114.015784.

2. Schneeberger K, Hagmann J, Ossowski S, Warthmann N, Gesing S, Kohlbacher O, Weigel D. Simultaneous alignment of short reads against multiple genomes. Genome Biol. 2009;10(9):98. https://doi.org/10.1186/gb-200910-9-r98.

3. Dilthey A, Cox C, Iqbal Z, Nelson MR, McVean G. Improved genome inference in the MHC using a population reference graph. Nat Genet. 2015;47(6):682-8. https://doi.org/10.1038/ng.3257.

4. Garrison E, Sirén J, Novak AM, Hickey G, Eizenga JM, Dawson ET, Jones W, Garg S, Markello C, Lin MF, Paten B, Durbin R. Variation graph toolkit improves read mapping by representing genetic variation in the reference. Nat Biotechnol. 2018;36(9):875-9. https://doi.org/10.1038/nbt.4227. 
5. Iqbal Z, Caccamo M, Turner I, Flicek P, McVean G. De novo assembly and genotyping of variants using colored de Bruijn graphs. Nat Genet. 2012;44(2):226-32. https://doi.org/10.1038/ng.1028.

6. Eggertsson HP, Jonsson H, Kristmundsdottir S, Hjartarson E, Kehr B, Masson G, Zink F, Hjorleifsson KE, Jonasdottir A, Jonasdottir A, Jonsdottir I, Gudbjartsson DF, Melsted P, Stefansson K, Halldorsson BV. Graphtyper enables population-scale genotyping using pangenome graphs. Nat Genet. 2017:49(11):1654-60. https://doi.org/10.1038/ ng.3964.

7. Sibbesen JA, Maretty L, Krogh A. Accurate genotyping across variant classes and lengths using variant graphs. Nat Genet. 2018;50(7):1054. https://doi.org/10.1038/s41588-018-0145-5.

8. Sirén J, Monlong J, Chang X, Novak AM, Eizenga JM, Markello C, Sibbesen J, Hickey G, Chang P-C, Carroll A, Haussler D, Garrison E, Paten B. Genotyping common, large structural variations in 5,202 genomes using pangenomes, the giraffe mapper, and the vg toolkit. bioRxiv. 2020. https://doi.org/10.1101/2020.12.04.412486.

9. Eggertsson HP, Kristmundsdottir S, Beyter D, Jonsson H, Skuladottir A, Hardarson MT, Gudbjartsson DF, Stefansson K, Halldorsson BV, Melsted P. GraphTyper2 enables population-scale genotyping of structural variation using pangenome graphs. Nat Commun. 2019;10(1):5402. https://doi.org/10.1038/s41467-019-13341-9. Number: 1 Publisher: Nature Publishing Group.

10. Danecek P, Auton A, Abecasis G, Albers CA, Banks E, DePristo MA, Handsaker RE, Lunter G, Marth GT, Sherry ST, McVean G, Durbin R, Group GPA. The variant call format and VCFtools. Bioinformatics. 2011;27(15):2156-8. https:// doi.org/10.1093/bioinformatics/btr330. Publisher: Oxford Academic.

11. Korn JM, Kuruvilla FG, McCarroll SA, Wysoker A, Nemesh J, Cawley S, Hubbell E, Veitch J, Collins PJ, Darvishi K, Lee C, Nizzari MM, Gabriel SB, Purcell S, Daly MJ, Altshuler D. Integrated genotype calling and association analysis of SNPs, common copy number polymorphisms and rare CNVs. 40(10):1253-60. https://doi.org/10.1038/ng. 237. Accessed 08-06-2021.

12. Maciuca S, Elias CdO, McVean G, lqbal Z. A natural encoding of genetic variation in a Burrows-Wheeler Transform to enable mapping and genome inference. In: Springer, editor. Proceedings of the 16th International Workshop on Algorithms in Bioinformatics; 2016. p. 222-33.

13. Valenzuela $D$, Norri T, Välimäki N, Pitkänen $E$, Mäkinen V. Towards pan-genome read alignment to improve variation calling. BMC Genomics. 2018;19(2):87. https://doi.org/10.1186/s12864-018-4465-8.

14. Ferragina P, Manzini G. Opportunistic data structures with applications. In: Proceedings 41 st Annual Symposium on Foundations of Computer Science. Redondo Beach, CA, USA: IEEE Comput. Soc; 2000. p. 390-8. https://doi.org/10. 1109/SFCS.2000.892127. http://ieeexplore.ieee.org/document/892127/.

15. Colquhoun RM, Hall MB, Lima L, Roberts LW, Malone KM, Hunt M, Letcher B, Hawkey J, George S, Pankhurst L, lqbal Z. Nucleotide-resolution bacterial pan-genomics with reference graphs. bioRxiv. 20202020-1112380378. https://doi.org/10.1 101/2020.11.12.380378. Publisher: Cold Spring Harbor Laboratory Section: New Results.

16. Bray NL, Pimentel H, Melsted P, Pachter L. Near-optimal probabilistic RNA-seq quantification. Nat Biotechnol. 2016;34(5):525-7. https://doi.org/10.1038/nbt.3519.

17. Ecma International: The JSON Data Interchange Syntax. Geneva. 2017. Ecma International. https://www.ecmainternational.org/publications-and-standards/standards/ecma-404/.

18. Ochola LI, Tetteh KKA, Stewart LB, Riitho V, Marsh K, Conway DJ. Allele frequency-based and polymorphism-versusdivergence indices of balancing selection in a new filtered set of polymorphic genes in Plasmodium falciparum. Mol Biol Evol. 2010;27(10):2344-51. https://doi.org/10.1093/molbev/msq119. Publisher: Oxford Academic.

19. Amambua-Ngwa A, Tetteh KKA, Manske M, Gomez-Escobar N, Stewart LB, Deerhake ME, Cheeseman IH, Newbold Cl, Holder AA, Knuepfer E, Janha O, Jallow M, Campino S, Maclnnis B, Kwiatkowski DP, Conway DJ. Population genomic scan for candidate signatures of balancing selection to guide antigen characterization in malaria parasites. PLoS Genet. 2012;8(11):1002992. https://doi.org/10.1371/journal.pgen.1002992.

20. The Pf3K Project 2015 Pilot Data Release 3. http://www.malariagen.net/data/pf3k-3. Accessed 27 Aug 2021.

21. Li H, Handsaker B, Wysoker A, Fennell T, Ruan J, Homer N, Marth G, Abecasis G, Durbin R, Subgroup GPDP. The Sequence Alignment/Map format and SAMtools. Bioinformatics. 2009;25(16):2078-9. https://doi.org/10.1093/ bioinformatics/btp352.

22. Miles A, lqbal Z, Vauterin P, Pearson R, Campino S, Theron M, Gould K, Mead D, Drury E, O'Brien J, Rubio VR, MacInnis B, Mwangi J, Samarakoon U, Ranford-Cartwright L, Ferdig M, Hayton K, Su X.-z., Wellems T, Rayner J, McVean G, Kwiatkowski D. Indels, structural variation, and recombination drive genomic diversity in Plasmodium falciparum. Genome Res. 2016;26(9):1288-99. https://doi.org/10.1101/gr.203711.115.

23. Richards JS, Beeson JG. The future for blood-stage vaccines against malaria. Immunol Cell Biol. 2009;87(5):377-90. https://doi.org/10.1038/icb.2009.27.

24. Barry $A E$, Arnott $A$. Strategies for designing and monitoring malaria vaccines targeting diverse antigens. Front Immunol. 2014;5:359. https://doi.org/10.3389/fimmu.2014.00359.

25. Otto TD, Böhme U, Sanders M, Reid A, Bruske El, Duffy CW, Bull PC, Pearson RD, Abdi A, Dimonte S, Stewart LB, Campino S, Kekre M, Hamilton WL, Claessens A, Volkman SK, Ndiaye D, Amambua-Ngwa A, Diakite M, Fairhurst RM, Conway DJ, Franck M, Newbold Cl, Berriman M. Long read assemblies of geographically dispersed Plasmodium falciparum isolates reveal highly structured subtelomeres. Wellcome Open Research. 2018;3:52. https:// doi.org/10.12688/wellcomeopenres.14571.1.

26. Borrell S, Trauner A, Brites D, Rigouts L, Loiseau C, Coscolla M, Niemann S, De Jong B, Yeboah-Manu D, Kato-Maeda M, Feldmann J, Reinhard M, Beisel C, Gagneux S. Reference set of Mycobacterium tuberculosis clinical strains: a tool for research and product development. PLOS ONE. 2019;14(3):1-12. https://doi.org/10.1371/journal. pone.0214088.

27. Chiner-Oms A, Berney M, Boinett C, González-Candelas F, Young DB, Gagneux S, Jacobs WR, Parkhill J, Cortes T, Comas I. Genome-wide mutational biases fuel transcriptional diversity in the Mycobacterium tuberculosis complex. Nat Commun. 2019;10(1):3994. https://doi.org/10.1038/s41467-019-11948-6.

28. Cole ST, Brosch R, Parkhill J, Garnier T, Churcher C, Harris D, Gordon SV, Eiglmeier K, Gas S, Barry CE, Tekaia F, Badcock K, Basham D, Brown D, Chillingworth T, Connor R, Davies R, Devlin K, Feltwell T, Gentles S, Hamlin N, Holroyd S, Hornsby T, Jagels K, Krogh A, McLean J, Moule S, Murphy L, Oliver K, Osborne J, Quail MA, 
Rajandream M-A, Rogers J, Rutter S, Seeger K, Skelton J, Squares R, Squares S, Sulston JE, Taylor K, Whitehead S, Barrell BG. Deciphering the biology of mycobacterium tuberculosis from the complete genome sequence. Nature. 1998;393(6685):537-44. https://doi.org/10.1038/31159.

29. Li H. Minimap2: pairwise alignment for nucleotide sequences. Bioinformatics. 2018;34(18):3094-100. https://doi.org/ 10.1093/bioinformatics/bty191. Publisher: Oxford Academic.

30. Langmead B, Salzberg SL. Fast gapped-read alignment with Bowtie 2. Nat Methods. 2012;9(4):357-9. https://doi. org/10.1038/nmeth.1923.

31. Büchler T, Ohlebusch E. An improved encoding of genetic variation in a Burrows-Wheeler transform. Bioinformatics. 2020;36(5):1413-9. https://doi.org/10.1093/bioinformatics/btz782. Publisher: Oxford Academic.

32. Crosnier C, Iqbal Z, Knuepfer E, Maciuca S, Perrin AJ, Kamuyu G, Goulding D, Bustamante LY, Miles A, Moore SC, Dougan G, Holder AA, Kwiatkowski DP, Rayner JC, Pleass RJ, Wright GJ. Binding of Plasmodium falciparum merozoite surface proteins DBLMSP and DBLMSP2 to human immunoglobulin M is conserved among Broadly diverged sequence variants. J Biol Chem. 2016;291(27):14285-99. https://doi.org/10.1074/jbc.M116.722074.

33. Gagie T, Manzini G, Sirén J. Wheeler graphs: a framework for BWT-based data structures. 698:67-78. https://doi.org/ 10.1016/j.tcs.2017.06.016. Accessed 04-08-2019.

34. The CRyPTIC consortium. A data compendium for M. tuberculosis antibiotic resistance (in preparation).

35. Li H, Feng $X$, Chu C. The design and construction of reference pangenome graphs with minigraph. Genome Biol. 2020;21 (1):265. https://doi.org/10.1186/s13059-020-02168-z.

36. Paten B, Eizenga JM, Rosen YM, Novak AM, Garrison E, Hickey G. Superbubbles, ultrabubbles, and cacti. J Comput Biol. 2018;25(7):649-63. https://doi.org/10.1089/cmb.2017.0251. Publisher: Mary Ann Liebert, Inc., publishers.

37. Pritt J, Chen N-C, Langmead B. FORGe: prioritizing variants for graph genomes. Genome Biol. 2018;19(1):220. https://doi.org/10.1186/s13059-018-1595-x.

38. Church DM, Schneider VA, Steinberg KM, Schatz MC, Quinlan AR, Chin C-S, Kitts PA, Aken B, Marth GT, Hoffman MM, Herrero J, Mendoza MLZ, Durbin R, Flicek P. Extending reference assembly models. Genome Biol. 2015;16(1): 13. https://doi.org/10.1186/s13059-015-0587-3.

39. Siren J, Välimäki N, Mäkinen V. [GCSA]indexing graphs for path queries with applications in Genome Research. IEEE/ACM Trans Comput Biol Bioinform. 2014;11(2):375-88. https://doi.org/10.1109/TCBB.2013.2297101.

40. Katoh K, Misawa K, Kuma K, Miyata T. MAFFT: a novel method for rapid multiple sequence alignment based on fast Fourier transform. Nucleic Acids Res. 2002;30(14):3059-66. https://doi.org/10.1093/nar/gkf436.

41. Huang W, Li L, Myers JR, Marth GT. ART: a next-generation sequencing read simulator. Bioinformatics. 2011;28(4): 593-4. https://doi.org/10.1093/bioinformatics/btr708.

42. Wick RR, Judd LM, Gorrie CL, Holt KE. Unicycler: resolving bacterial genome assemblies from short and long sequencing reads. PLoS Comput Biol. 2017;13(6):1005595. https://doi.org/10.1371/journal.pcbi.1005595. Publisher: Public Library of Science.

43. Koren S, Walenz BP, Berlin K, Miller JR, Bergman NH, Phillippy AM. Canu: scalable and accurate long-read assembly via adaptive k-mer weighting and repeat separation. Genome Res. 2017;27(5):722-36. https://doi.org/10.1101/gr. 215087.116. Company: Cold Spring Harbor Laboratory Press Distributor: Cold Spring Harbor Laboratory Press Institution: Cold Spring Harbor Laboratory Press Label: Cold Spring Harbor Laboratory Press Publisher: Cold Spring Harbor Lab.

44. Hunt M, Silva ND, Otto TD, Parkhill J, Keane JA, Harris SR. Circlator: automated circularization of genome assemblies using long sequencing reads. Genome Biol. 2015;16(1):294. https://doi.org/10.1186/s13059-015-0849-0.

45. Kolmogorov M, Yuan J, Lin Y, Pevzner PA. Assembly of long, error-prone reads using repeat graphs. Nat Biotechnol. 2019;1:540-6. https://doi.org/10.1038/s41587-019-0072-8.

46. Carver TJ, Rutherford KM, Berriman M, Rajandream M-A, Barrell BG, Parkhill J. ACT: the Artemis comparison tool. Bioinformatics. 2005;21(16):3422-3. https://doi.org/10.1093/bioinformatics/bti553. https://academic.oup.com/ bioinformatics/article-pdf/21/16/3422/573752/bti553.pdf.

47. Walker BJ, Abeel T, Shea T, Priest M, Abouelliel A, Sakthikumar S, Cuomo CA, Zeng Q, Wortman J, Young SK, Earl AM. Pilon: an integrated tool for comprehensive microbial variant detection and genome assembly improvement. PLoS ONE. 2014;9(11):112963. https://doi.org/10.1371/journal.pone.0112963. Publisher: Public Library of Science.

48. Li H. Aligning sequence reads, clone sequences and assembly contigs with BWA-MEM. 2013. http://arxiv.org/abs/ 1303.3997.

49. Kurtz S, Phillippy A, Delcher AL, Smoot M, Shumway M, Antonescu C, Salzberg SL. Versatile and open software for comparing large genomes. Genome Biol. 2004;5(2):12. https://doi.org/10.1186/gb-2004-5-2-r12.

50. Šošić M, Šikić M. Edlib: a C/C ++ library for fast, exact sequence alignment using edit distance. Bioinformatics. 2017;33(9):1394-5. https://doi.org/10.1093/bioinformatics/btw753. Publisher: Oxford Academic.

51. Hunt MH, Letcher B, Malone KM, Nguyen G, Hall MB, Colquhoun RM, Lima L, Schatz M, Ramakrishnan S, CRyPTIC consortium, Iqbal Z. Minos: graph adjudication and joint genotyping of cohorts of bacterial genomes (in preparation).

52. Letcher B, Hunt MH, Iqbal Z. Gramtools Github Software Repository. https://github.com/iqbal-lab-org/ gramtools. Accessed 10 August 2021.

53. Letcher B, Hunt MH, Iqbal Z. Gramtools: Genome Graph Genotyper. https://doi.org/10.5281/zenodo. 5176372. Accessed 10 August 2021.

54. Köster J, Rahmann S. Snakemake'a scalable bioinformatics workflow engine. Bioinformatics. 2012;28(19):2520-2. https://doi.org/10.1093/bioinformatics/bts480. Publisher: Oxford Academic.

\section{Publisher's Note}

Springer Nature remains neutral with regard to jurisdictional claims in published maps and institutional affiliations. 